\title{
Integrated generation-consumption dispatch based on compensation mechanism considering demand response behavior
}

\author{
Yumin ZHANG ${ }^{1}$, Xueshan HAN ${ }^{1}$, Li ZHANG ${ }^{1}$, Bo XU ${ }^{2}$, \\ Mingqiang WANG ${ }^{1}$, Ming YANG ${ }^{1}$
}

\begin{abstract}
Demand response (DR) is important to account for behaviors of the demand side to yield an optimal dispatch result. However, it is difficult for energy suppliers to collect customers' private information unless there is an incentive mechanism for customers to do so. Therefore, this paper proposes a new integrated generation-consumption dispatch based on compensation mechanism considering DR behavior. Firstly, in light of the dayahead load forecast data, we deduce the utility function model of different customers. By subtracting generating units' operation cost from consumers' total utility, the dispatch model have a decentralized demand participant
\end{abstract}

CrossCheck date: 23 November 2017

Received: 22 August 2017 / Accepted: 23 November 2017/Published online: 7 February 2018

(C) The Author(s) 2018. This article is an open access publication

$\square$ Yumin ZHANG

zymcth@126.com

Xueshan HAN

xshan@sdu.edu.cn

Li ZHANG

yzhangli@sdu.edu.cn

Bo XU

xubo@mail.tsinghua.edu.cn

Mingqiang WANG

wang0367@hotmail.com

Ming YANG

myang@sdu.edu.com

1 Key Laboratory of Power System Intelligent Dispatch and Control of Ministry of Education, Shandong University, Jinan 250061, China

2 State Key Laboratory of Control and Simulation of Power Systems and Generation Equipment, Department of Electrical Engineering, Tsinghua University, Beijing 100084, China structure based on this utility function. The utility function is used to describe consumers' preferences and energy consumption behaviors. Secondly, an effective compensation mechanism is designed to ensure customers to select the level of compensation appropriate to their willingness to curtail load. Finally, a new dispatch model is proposed that incorporates the DR compensation mechanism into the generation-consumption dispatch model. The new model can improve the interaction of generation and consumption, and benefit both the energy supplier and its customers. The proposed model is piecewise linearized and solved by a mixed-integer linear programming method. It is tested on a six-bus system and the IEEE 118-bus system. Simulation results show that the proposed model can realize both maximum social welfare and Pareto optimal results.

Keywords Compensation mechanism, Demand response, Demand utility function, Integrated generationconsumption dispatch

\section{Introduction}

With the high penetration of renewable generation and the increasing demand, the uncertainties in power systems are gradually increasing [1]. Therefore, more controllable devices are required to accommodate the uncertainties [2]. It is insufficient to rely only on the controllable generation resources in power system. Although electric vehicles [3] and storage [4] act as controllable resources, their excessive investment cost makes them difficult to obtain on a large-scale [5-7]. The more direct, effective, and economical way may be to guide customers to consume electricity rationally and efficiently $[8,9]$. Thus, the role of 
demand response (DR) becomes more and more prominent [10].

Customers' behaviors will affect units' dispatch strategy, and enhancing the interaction between an energy supplier and its customers can eliminate the energy imbalances in power systems [11]. Analyzing the response behavior of different types of consumers is the basis for designing effective DR strategies to match the conventional generation dispatch. Meanwhile, designing reasonable market mechanisms, including appropriate compensation scheme for customers providing DR, is essential to ensure that DR is available to assist the safe and economic operation of power system.

An energy supplier designs a DR compensation scheme in order to acquire reliable resources that maybe scheduled in a timely manner, helping to cope with an emergency shortage of generation. Although the mechanism can be better designed using optimization methods $[12,13]$, what counts more is whether it is designed reasonably and executed accurately by the customers. A reasonable scheme can only be developed if sufficient information can be obtained, but in practice there exists an informational asymmetry between the energy supplier and its customers, since a customer's private information (e.g. outage cost and preference parameters) is unknown to the energy supplier and other customers. In general, this information is difficult to obtain accurately from commercial customers due to their confidentiality requirements for strategic purposes [14]. Moreover, some customers wishing to maximize their own benefit may lie to energy suppliers $[15,16]$. These factors can lead to low efficiency of the DR mechanism.

To solve this problem, [17] proposes mechanism design theory, and it encourages the participants to disclose their true value of outage cost, willingness and other private information by combining incentive compatibility and informational efficiency. Reference [18] introduces a continuous customer type parameter into the customer cost function, and applies mechanism design principles to formulate a model interruptible load management (ILM) contract with incentive compatibility, which is suitable for different types of customers. Reference [19] presents an improved approach to estimate the customer type and the customer outage cost function by using historical demand side management (DSM) data, and formulates the ILM contract model with a discrete customer type parameter. All show that the critical issue is how to give effective financial compensation to customers to encourage helpful energy consumption behavior and alleviate stress on the power grid through load curtailment. Mechanism design seems a promising way to solve the problem.

In China, an electricity market on the power generation side was created by separating power plants from the power network. Price competition is still not perfect in this market, and dispatch operations and electricity sales are still being integrated. Including reasonable DR mechanisms into the system dispatch model is an effective approach to improve economic efficiency and increase system security. In [1, 20, 21] time-of-use (TOU) pricing and interruptible load (IL) have been integrated into the conventional generation dispatch model and they can reduce cost and improve the accommodation of renewable energy. In [22, 23] the authors discuss the impact of price changes on demand elasticity and propose that unit commitment (UC) can be determined by minimum cost rather than optimal power flow (OPF) in the generation dispatch model. In [24], the author analyzes the impact of pricebased DR on market clearing and locational marginal prices (LMPs) in a power system using network-constrained unit commitment (NCUC).

However, these studies are all based on the following assumptions: 1) demand is treated as a fixed value at each time (inelastic demand); 2) customers' benefit functions are constant or linear functions of price, and their preferences are expressed solely in the form of constraints; 3) the integrated generation-consumption dispatch problem is converted from a social welfare maximization problem to an equivalent generation cost minimization problem. The market price is determined only by the marginal cost of generators. Additionally, it is considered that this price equals the consumers' marginal utility. These assumptions help to quantify the model, however, they may not reflect the impacts of the demand side on market price setting, which may cause price spikes, and they fail to explain the actual DR behavior and reflect customers' true utility. It is important that market pricing is co-determined from a common decision between the supply side and the demand side. Thus, DR mechanisms that do not consider users' utility would fail to incentivize the initiative of customers.

The novel contribution of this paper, based on the electricity market in China [25], is an integrated generation-consumption dispatch model considering DR behavior based on mechanism design principles. Firstly, in light of the day-ahead load forecast data, we can deduce the utility function of different customers. The utility function is used to describe consumers' preferences and energy consumption behaviors. Secondly, by subtracting units' operational cost from consumers' total utility, a new integrated generation-consumption dispatch model is formulated with decentralized demand participation. The new model improves the interaction of suppliers and their customers, which can benefit both of them. Thirdly, based on the above UC results, taking into account the behaviors of users and security threats of the system, an effective compensation mechanism is designed to ensure customers 
select a compensation scheme according to their true willingness to curtail of load. Finally, a new dispatch model is formed by incorporating the DR compensation mechanism into the generation-consumption dispatch model, which co-optimizes DR and generation-consumption dispatch decisions. The new model can realize maximum social welfare with Pareto optimality for which the customers' marginal utility is equal to the marginal cost of generators.

The remainder of the paper is organized as follows. Section 2 describes the customer behavior and the mathematical model of the proposed compensation mechanism. Section 3 proposes the integrated generation-consumption dispatch model considering the demand utility function and the DR compensation mechanism integration. Section 4 gives the detailed method to obtain optimal solutions using the model. Section 5 demonstrates and discusses numerical results obtained for a six-bus system and the IEEE 118-bus system. Finally, Section 6 concludes this work. Appendix A gives a simple example to illustrate a derivation required for the analysis $\theta$. Appendix B presents some details about the mathematical formulation of compensation mechanism.

\section{Customer behavior and compensation mechanism}

\subsection{Customer behavior}

Influenced by the factors such as the time, environment, national policies, etc., the electricity demand of each customer is different, therefore their response to certain incentives differs. According to the principle of microeconomics, a quadratic utility function is adopted to represent customers' preferences and consumption behaviors [26]:

$U\left(\alpha_{j t}, D_{j t}\right)= \begin{cases}\alpha_{j t} D_{j t}-\frac{1}{2 \beta_{j t} D_{j t}^{2}} & 0 \leq D_{j t} \leq \frac{\alpha_{j t}}{\beta_{j t}} \\ \frac{\alpha_{j t}^{2}}{2 \beta_{j t}} & D_{j t} \geq \frac{\alpha_{j t}}{\beta_{j t}}\end{cases}$

The marginal utility is then

$$
\begin{aligned}
M U\left(\alpha_{j t}, D_{j t}\right) & =\frac{\partial U\left(\alpha_{j t}, D_{j t}\right)}{\partial D_{j t}} \\
& = \begin{cases}-\beta_{j t} D_{j t}+\alpha_{j t} & 0 \leq D_{j t} \leq \frac{\alpha_{j t}}{\beta_{j t}} \\
0 & D_{j t} \geq \frac{\alpha_{j t}}{\beta_{j t}}\end{cases}
\end{aligned}
$$

In (1) and (2), $D_{j t}$ is the electricity demand of customer $j$ in period $t ; \alpha_{j t}$ is a parameter characterizing the demand preference of customer $j$ in period $t ; \beta_{j t}$ is the saturation point of the utility of customer $j$ in period $t$. The greater is $\alpha_{j t}$, the greater is the customer's saturation electricity demand. The greater is $\beta_{j t}$, the lower is the customer's saturation electricity demand [27]. It can be seen that the utility function is concave. The customer's marginal utility increases as the demand increases before reaching the saturation point, and is assumed to be constant afterwards. Assuming the customer is rational, his or her demand will naturally maximize individual utility. Consequently, $\bar{D}$ reflects the customer's demand of maximum utility. At this optimal point, the marginal utility is zero.

$\frac{\alpha_{j t}}{\beta_{j t}}=\bar{D}_{j}$

Therefore, (1) can be replaced by:

$U\left(\alpha_{j t}, D_{j t}\right)= \begin{cases}\alpha_{j t} D_{j t}-\frac{1}{2 \beta_{j t} D_{j t}^{2}} & 0 \leq D_{j t} \leq \bar{D}_{j} \\ \frac{\alpha_{j t}^{2}}{2 \beta_{j t}} & D_{j t} \geq \bar{D}_{j}\end{cases}$

Figure 1 illustrates the impacts of marginal utility on customers' energy consumption behaviors. A part of the demand is necessary for the customers to maintain their basic productivity, and this part is perfectly inelastic and is defined as the taking load. Therefore, the responsive load part has a maximum range. To accurately describe the market participation behavior of customers and to better reflect the potential for customers to participate in DR, we define the ratio $D R L_{j t}$ of the DR amount and the original demand $D_{j t}^{\mathrm{o}}$ of customer $j$ in period $t$ as the DR level of the customer. Hence, the $D R L_{j t}$ is in the range $[0,1] . D R L_{j t}=0$ represents the case in which customer $j$ in period $t$ is not participating in DR.

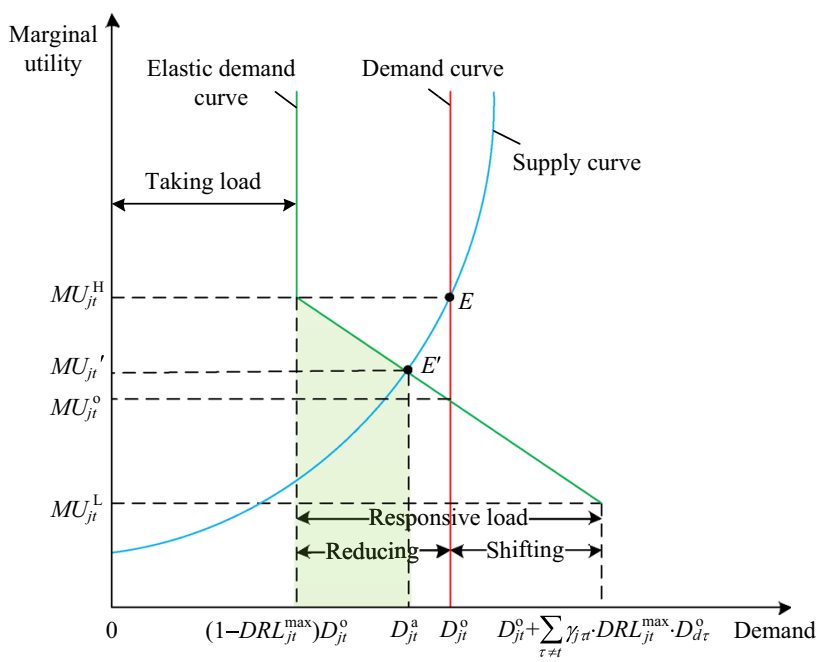

Fig. 1 Impact of marginal utility on DR level 
$D R L_{j t}=\frac{D_{j t}^{\mathrm{o}}-D_{j t}^{\mathrm{a}}}{D_{j t}^{\mathrm{o}}}$

where $D_{j t}^{\mathrm{a}}$ is the actual load demand of customer $j$ in period $t$ after the DR participation.

As shown in Fig. 1, the marginal utility of customer $j$ in period $t$ during the peak periods is $M U_{j t}^{\mathrm{H}}$. If the demand amount is reduced to $D_{j t}^{\mathrm{a}}$ as the marginal utility decreases, the market will also fall to a new equilibrium $E^{\prime}$. The unmet partial load during the peak periods will shift to the off-peak periods because their relative marginal utility increases during the off-peak periods $M U_{j t}^{\mathrm{L}}$. Therefore, the demand will increase to $D_{j t}^{\mathrm{o}}+\sum_{\tau \neq t} \gamma_{j \tau t} \cdot D R L_{j \tau}^{\max } \cdot D_{j \tau}^{\mathrm{o}}$, where $D R L_{j t}^{\max }$ is the maximum DR level of customer $j$ in period $t$ and $\gamma_{j \tau t}$ is the load-shifting rate of customer $j$ from time $t$ to time $\tau$. This reflects that customers' initiative leads to behaviors that are driven by their marginal utility.

This paper only focuses on load curtailment, the case of load-shifting is beyond the scope of this paper, namely $\gamma_{j \tau t}=0$. Hence the demand of customer $j$ in period $t$ when participating in DR is in the range as shown in (6).

$\left(1-D R L_{j t}^{\max }\right) D_{j t}^{\mathrm{o}} \leq D_{j t}^{\mathrm{a}} \leq D_{j t}^{\mathrm{o}}$

Since the marginal utility curve passes through the two points $\quad\left(\left(1-D R L_{j t}^{\max }\right) D_{j t}^{\mathrm{o}}, M U_{j t}^{\mathrm{H}}\right), \quad\left(D_{j t}^{\mathrm{o}}, M U_{j t}^{\mathrm{o}}\right), \quad$ the parameters of marginal utility (2) can be derived as:

$\alpha_{j t}=\frac{M U_{j t}^{\mathrm{H}}+\left(D R L_{j t}^{\max }-1\right) \cdot M U_{j t}^{\mathrm{o}}}{D R L_{j t}^{\max }}$

$\beta_{j t}=\frac{M U_{j t}^{\mathrm{H}}-M U_{j t}^{\mathrm{o}}}{D R L_{j t}^{\max } \cdot D_{j t}^{\mathrm{o}}}$

Here, for the sake of simplicity, we shall assume that the customer gross surplus for the taking load part is a constant and thus it can be neglected in the utility function. Therefore, the utility is limited to the responsive load part, namely from $\left(1-D R L_{j t}^{\max }\right) D_{j t}^{\mathrm{o}}$ to $D_{j t}^{\mathrm{a}}$, as shown in the green shaded area in Fig. 1 . Assuming that $D_{j t} \leq \bar{D}_{j}$, we have

$$
\begin{aligned}
U\left(\alpha_{j t}, D_{j t}\right)= & \int_{\left(1-D R L_{j t}\right) \cdot D_{j t}^{\mathrm{o}}}^{D_{j t}^{\mathrm{a}}}\left(-\beta_{j t} \tilde{D}-\alpha_{j t}\right) \mathrm{d} \tilde{D} \\
= & \alpha_{j t} D_{j t}^{\mathrm{a}}-\frac{1}{2} \beta_{j t}\left(D_{j t}^{\mathrm{a}}\right)^{2}-\alpha_{j t}\left(1-D R L_{j t}^{\mathrm{max}}\right) D_{j t}^{\mathrm{o}} \\
& \quad-\frac{1}{2} \beta_{j t}\left[\left(1-D R L_{j t}^{\max }\right) D_{j t}^{\mathrm{o}}\right]^{2}
\end{aligned}
$$

Note that (9) is a quadratic utility function which is difficult to use in analysis due to its nonlinearity. To circumvent this problem, we can use a piece-wise linear approximation to obtain the formulation shown in (11), which is suitable for mixed-integer linear programming (MILP).

$U\left(\alpha_{j t}, D_{j t}\right)=\sum_{k=1}^{M} s_{j k t} D_{j k t}^{\mathrm{a}}$

$\left\{\begin{array}{l}s_{j k t}=\frac{U\left(\alpha_{j t}, D_{j k t}\right)-U\left(\alpha_{j t}, D_{j, k-1, t}\right)}{D_{j k t}-D_{j, k-1, t}} \\ 0 \leq D_{j k t}^{\mathrm{a}} \leq D_{j k t}-D_{j, k-1, t} \\ D_{j t}^{\mathrm{a}}=\left(1-D R L_{j t}^{\max }\right) D_{j t}^{\mathrm{o}}+\sum_{k=1}^{M} D_{j k t}^{\mathrm{a}} \\ D_{j k t}=\left(1-D R L_{j t}^{\max }\right) D_{j t}^{\mathrm{o}}+\frac{k}{M} \cdot D R L_{j t}^{\max } \cdot D_{j t}^{\mathrm{o}}\end{array}\right.$

where $s_{j k t}$ is the marginal utility at segment $k$ of customer $j$ in period $t ; D_{j k t}$ and $D_{j k t}^{\mathrm{a}}$ are respectively the load demand and the actual load demand at segment $k$ of customer $j$ in period $t$ in the linearized DR formulation; $M$ is the set of segments. A similar argument applies to the case when $D_{j t} \geq \bar{D}_{j}$.

\subsection{Compensation mechanism}

From the perspective of maximizing social welfare, the balance between supply-side and demand-side responses must be the result of trading off generation cost and demand utility. Therefore, if DR is integrated into the conventional generation dispatch model, it will be necessary to investigate the impact of demand utility on the changes of customers' energy consumption behaviors. The expected generation schedule will be realized by compensating the changes of the demand utility. For example, during peak periods, a reasonable compensatory mechanism can motivate customers to reduce load voluntarily. A compensation mechanism should determine the optimal amount of DR and optimal incentive fees to encourage the amount of DR that each customer is willing to curtail.

The cost of demand reduction is modelled with two key parameters: the preference parameter $\theta_{j}$ of the willingness of customer $j$ to curtail and the amount of his load curtailment $d r_{j t}$ (MW) in period $t$, such that the outage cost $C\left(\theta_{j}, d r_{j t}\right)$ of the customer $\theta_{j}$ for curtailing $d r_{j t}$ is:

$C\left(\theta_{j}, d r_{j t}\right)=K_{1}\left(d r_{j t}\right)^{2}+K_{2}\left(1-\theta_{j}\right) \cdot d r_{j t}$

where $\theta_{j}$ is used to characterize customers' preference for curtailment probabilistically, and we assume this "preference parameter" $\theta_{j}$ possesses a uniform probability distribution $f\left(\theta_{j}\right)$ in the interval $[0,1]$. It sorts the customers from the least willing to the most willing to curtail load. $K_{1}$ and $K_{2}$ are the quadratic and linear term coefficients, respectively, of the outage cost function. These parameters are private customer information that is unknown to the 
supply-side. They are all positive, and they can be estimated using existing data. For the sake of simplicity, this paper assumes that $K_{1}=0.5$ and $K_{2}=1$ [19]. Appendix A gives a simple example to illustrate the derivation of the assumed values of $K_{1}, K_{2}$ and all the $\theta_{j}$.

If rational customers do not receive any compensation for their demand reduction, they will not choose to participate in the DR program. Such customers will self-select the amount of demand curtailment $d r_{j t}$ based on the monetary compensation $y$ offered by the energy supplier in order to maximize their utility. Hence, the customers' benefit $U^{\mathrm{d}}(\cdot)$ is defined as the amount of monetary compensation they received from the energy supplier minus their outage cost due to the curtailment.

$U^{\mathrm{d}}\left(\theta_{j}, d r_{j t}, y\right)=y-C\left(\theta_{j}, d r_{j t}\right)$

When the power network suffers from peak load or emergencies, LMPs will soar, which means it is expensive for an energy supplier to provide electricity to the customers at those locations. In order to maximize their own interests, the energy supplier will make a trade-off between the cost of providing electricity to the customer at each location and the cost of curtailing the customer. We define $V^{\mathrm{s}}\left(L_{j}, d r_{j t}\right)$ as the value of not delivering power to customer $j$ in period $t$. Hence, the energy suppliers' benefit $U^{\mathrm{s}}$ generated by the load curtailment of customer $j$ can be expressed as:

$U^{\mathrm{s}}\left(L_{j}, d r_{j t}, y\right)=V^{\mathrm{s}}\left(L_{j}, d r_{j t}\right)-y=L_{j} \cdot d r_{j t}-y$

where $L_{j}$ is parameterized value of not providing electricity to customer $j$ at a specific location. Each customer has a fixed value of $L_{j}$ since it cannot move and change its locational value. It also reflects the weak influence of customer location and the contribution of load curtailment for the grid. It can be obtained using optimal power flow routines [28].

The energy supplier will have a subjective estimate for each customer preference parameter $\theta_{j}$. The DR function $D R\left(d r_{j t}, L_{j}\right)$ illustrates how much load curtailment is obtained from customer preference parameter $\theta_{j}$ at location $L_{j}$. The compensation function $Y\left(d r_{j t}\right)$ indicates how much the energy supplier is willing to pay the DR participants for their curtailment.

The aim of designing the DR compensation mechanism is to calculate the optimal compensation fee for a customer to provide some amount megawatt of load curtailment, so as to improve security of the power grid. It is a mathematical optimization problem, which maximizes the total utility for DR. The objective function is:

$$
\begin{aligned}
\max _{D R(\cdot), Y(\cdot)} & E_{\theta}\left(U^{\mathrm{s}}\left(D R_{j t}\left(\theta_{j}, L_{j}\right), Y\left(D R_{j t}\left(\theta_{j}, L_{j}\right)\right), L_{j}\right)\right. \\
& \left.\quad+U^{\mathrm{d}}\left(D R_{j t}\left(\theta_{j}, L_{j}\right), Y\left(D R_{j t}\left(\theta_{j}, L_{j}\right)\right), \theta_{j}\right)\right) \\
= & \left(V^{\mathrm{s}}\left(L_{j}, D R_{j t}\right)-Y\left(D R_{j t}\right)\right)+\left(Y\left(D R_{j t}\right)-C\left(\theta_{j}, D R_{j t}\right)\right) \\
= & V^{\mathrm{s}}\left(L_{j}, D R_{j t}\right)-C\left(\theta_{j}, D R_{j t}\right)
\end{aligned}
$$

Both energy supplier and customer are assumed to be rational. The energy supplier will only provide compensation for customers at more critical locations where it can reduce the threat to security of the power grid by curtailing some amount of load. As discussed above, customers will not curtail load unless there is a positive incentive for them. Thus both supplier and customer are subject to the individual rational constraints as (16), (17) and to the incentive compatibility constraint as (18) [19].

$$
\begin{gathered}
U^{\mathrm{s}}\left(D R_{j t}\left(\theta_{j}, L_{j}\right), Y\left(D R_{j t}\left(\theta_{j}, L_{j}\right)\right), L_{j}\right) \geq 0 \\
U^{\mathrm{d}}\left(D R_{j t}\left(\theta_{j}, L_{j}\right), Y\left(D R_{j t}\left(\theta_{j}, L_{j}\right)\right), \theta_{j}\right) \geq 0 \\
\quad U^{\mathrm{d}}\left(D R_{j t}\left(\theta_{j}, L_{j}\right), Y\left(D R_{j t}\left(\theta_{j}, L_{j}\right)\right), \theta_{j}\right) \\
\geq U^{\mathrm{d}}\left(D R_{j t}\left(\tilde{\theta}_{j}, L_{j}\right), Y\left(D R_{j t}\left(\tilde{\theta}_{j}, L_{j}\right)\right), \theta_{j}\right)
\end{gathered}
$$

Equation (18) illustrates that if a customer $\theta_{j}$ reports his information $\tilde{\theta}_{j}$ wrongly, his benefit will not be at the maximum, so it is not wise for the customer to lie. Hence, (18) can prevent customers from lying about $\theta_{j}$, and it ensures customers select their compensation scheme according to their true willingness to provide DR.

The diagram in Fig. 2 illustrates the principle of the compensation mechanism.

As mentioned above, the decision variables $D R(\cdot), Y(\cdot)$ of the proposed compensation mechanism are also the functions of the customer preference parameter $\theta_{j}$ and the location $L_{j}$, and they are given by (19) and (20). Their detailed mathematical derivations are given in Appendix B.

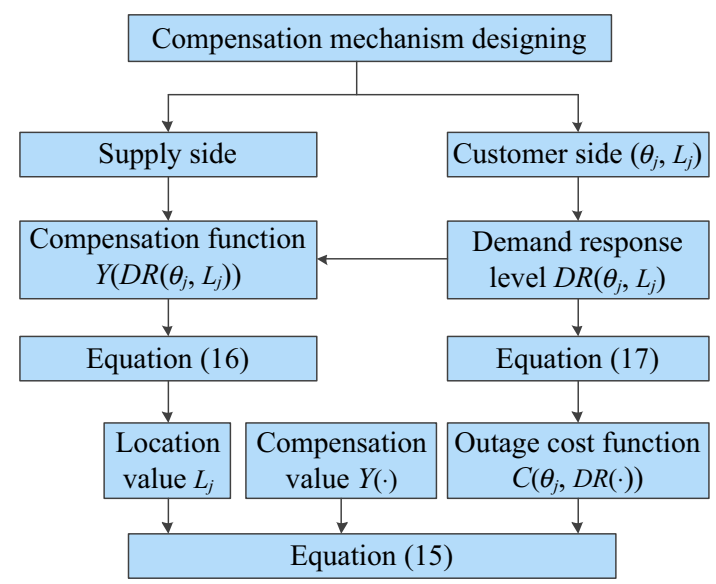

Fig. 2 Principle diagram of the compensation mechanism 


$$
\begin{aligned}
& D R_{j t}\left(\theta_{j}, L_{j}\right)= \begin{cases}0 & 0 \leq \theta_{j}<1-\frac{L_{j}}{2} \\
2 \theta_{j}+L_{j}-2 & 1-\frac{L_{j}}{2} \leq \theta_{j}<1\end{cases} \\
& Y\left(D R_{j t}\left(\theta_{j}, L_{j}\right)\right)=\left\{\begin{array}{lr}
0 & 0 \leq \theta_{j}<1-\frac{L_{j}}{2} \\
\theta_{j}^{2}-2 \theta_{j}+2 \theta_{j} L_{j}+\frac{3}{4} L_{j}^{2}-2 L_{j}+1 & \\
1-\frac{L_{j}}{2} \leq \theta_{j}<1
\end{array}\right.
\end{aligned}
$$

\section{Mathematical formulation}

\subsection{Integrated generation-consumption dispatch model}

Each individual user's behavior is assumed to be independent. Customers will make various responses to a given price according to their own utility maximization. But the individual optimal choice may not be equal to the social optimal choice. In order to unify individual optimal choice and social optimal choice, we propose the following optimization problem.

$\max _{P_{g t}, u_{g t}} F=\max \sum_{t=1}^{T}\left[\sum_{j=1}^{D} U\left(\alpha_{j t}, D_{j t}\right)-\sum_{g=1}^{G}\left(C_{g t} u_{g t}+S_{g t} v_{g t}\right)\right]$

$u_{g t}-u_{g, t-1}-v_{g t} \leq 0 \quad \forall g \in G, \forall t \in T$

$\sum_{g=1}^{G} P_{g t}=\sum_{j=1}^{D} D_{j t}^{\mathrm{o}} \quad \forall g \in G, \forall t \in T$

$u_{g t} P_{g}^{\min } \leq P_{g t} \leq u_{g t} P_{g}^{\max } \quad \forall g \in G, \forall t \in T$

$P_{g t}-P_{g, t-1} \leq r_{g}^{\mathrm{up}} u_{g, t-1}+P_{g}^{\max }\left(1-u_{g, t-1}\right) \quad \forall g, \forall t$

$P_{g, t-1}-P_{g t} \leq r_{g}^{\mathrm{dn}} u_{g t}+P_{g}^{\max }\left(1-u_{g t}\right) \quad \forall g, \forall t$

$\begin{cases}\sum_{t=1}^{T_{g}^{\mathrm{on}}}\left(1-u_{g t}\right)=0 & g=1,2, \ldots, G \\ t+X_{\mathrm{g}}^{\mathrm{on}}-1 & \\ \sum_{d=t}^{\mathrm{on}} u_{g d} \geq X_{g}^{\mathrm{on}}\left(u_{g t}-u_{g, t-1}\right) & g=1,2, \ldots, G ; t=T_{g}^{\mathrm{on}}, \ldots, T-X_{\mathrm{g}}^{\mathrm{on}}+1 \\ \sum_{d=t}^{T}\left[u_{g d}-\left(u_{g t}-u_{g, t-1}\right)\right] \geq 0 & g=1,2, \ldots, G ; t=T-X_{\mathrm{g}}^{\mathrm{on}}+2, \ldots, T\end{cases}$

$$
\begin{cases}\sum_{t=1}^{T_{g}^{\text {off }}} u_{g t}=0 & g=1,2, \ldots, G \\ t+X_{g}^{\text {off }}-1 & \\ \sum_{d=t}^{T}\left(1-u_{g d}\right) \geq X_{g}^{\text {off }}\left(u_{g, t-1}-u_{g t}\right) & g=1,2, \ldots, G ; t=T_{g}^{\text {off }}+1, \ldots, T-X_{\mathrm{g}}^{\text {off }}+1 \\ \sum_{d=t}^{T}\left[1-u_{g d}-\left(u_{g, t-1}-u_{g t}\right)\right] \geq 0 & g=1,2, \ldots, G ; t=T-X_{\mathrm{g}}^{\text {off }}+2, \ldots, T\end{cases}
$$

$$
\begin{aligned}
& \sum_{g=1}^{G_{b}} P_{g t}-\sum_{j=1}^{D_{b}} D_{j t}^{\mathrm{o}}=\sum_{j} B_{i j}\left(\theta_{i t}-\theta_{j t}\right) \quad \forall b, \forall t \\
& -F_{l}^{\max } \leq B_{i j}\left(\theta_{i t}-\theta_{j t}\right) \leq F_{l}^{\max } \quad \forall l, \forall t \\
& -\pi \leq \theta_{b t} \leq \pi \quad \forall b, \forall t \\
& \theta_{\mathrm{refb}, t}=0 \quad \forall t
\end{aligned}
$$

Equation (21) is subject to (4), (10) and (11) as well. The optimization goal (21) is to maximize the social welfare, in which the first term on the right-hand side represents the aggregate utilities of all customers, and the last two terms represent the unit commitment cost and the startup cost of thermal units. $C_{g t}=a_{g} P_{g t}^{2}+b_{g} P_{g t}+c_{g}$ is quadratic production cost function, where $a_{g}, b_{g}, c_{g}$ are the cost coefficients and $P_{g t}$ is power output of thermal unit $g$ in period $t . u_{g t}$ is the binary decision variable: on/off status of unit $g$ in period $t$, " 1 " if the generator is on, " 0 " otherwise. $v_{g t}$ is the binary decision variable: " 1 " if unit $g$ is started up in period $t$, " 0 " otherwise. $T$ is the set of scheduling periods. $D$ is set of loads. $G$ is set of thermal units. $S_{g t}=$ $K_{g}+B_{g}\left[1-\exp \left(-X_{g t}^{\text {off }} / \tau_{g}\right)\right]$ is a typical exponential startup cost function, for convenient analysis, these can be linearized using the piecewise linearization method with three segments [29-32].

Equation (22) gives the start-up constraints of thermal units. Equation (23) depicts the supply-demand balance requirement of the system, associate the dual variable $\lambda_{t}$ with (23), namely the Lagrangian multiplier, which is also the market clearing price (MCP) of the system. Equation (24) gives the generation capacity limits of thermal units. $P_{g}^{\max }$ and $P_{g}^{\min }$ are the maximum/minimum power output of thermal unit $g$, respectively. Equations (25) and (26) are the ramping up and down rate limits of thermal units, where $r_{g}^{\text {up }}$ and $r_{g}^{\mathrm{dn}}$ are the ramp-up/ramp-down rate for thermal unitg. Equations (27) and (28) describe the linearized minimum up-time and down-time constraints [29-32], where $T_{g}^{\text {on }}$ and $T_{g}^{\text {off }}$ are the minimal on/off hour of thermal unit $g$ ( 1 is on and 0 is off); $X_{g}^{\text {on }}$ and $X_{g}^{\text {off }}$ are the number of periods unit $g$ has been online/offline prior to the first period of the time span (end of period 0 ). This formulation can be converted into a MILP problem. Equation (29) represents the power balance equations for each node, where $\theta_{i t}$ is the phase angle of bus $i$ in period $t ; D_{b}$ is set of loads in bus $b ; G_{b}$ is set of thermal units in bus $b$. Equation (30) gives the network power flow limits $F_{l}^{\max }$ on transmission lines $l$, associate the dual variables $\pi_{l t}^{+}$and $\pi_{l t}^{-}$ with (30). $B_{i j}$ is element in row $i$ and column $j$ of DC power flow matrix. Equation (31) describes the upper and lower limits of the phase angle $\theta_{b t}$ and (32) represents the reference phase angle $\theta_{\text {refb }, t}$. 
After solving the model (21)-(32), the LMPs are calculated in terms of the dual variables:

$L M P_{b t}=\lambda_{t}+\sum_{l}\left(\pi_{l t}^{+}-\pi_{l t}^{-}\right)$

\subsection{Compensation mechanism in integrated model}

This section proposes an integrated generation-consumption dispatch model to calculate unit commitment decisions incorporating DR. The focus is on maximizing social welfare by including the benefits and costs of customers participating in DR (15) into the decision-making. The details are as follows.

$$
\begin{aligned}
& \max _{P_{g t}, D R_{j t}(\cdot), Y(\cdot)} F=\sum_{t=1}^{T}\left[\sum _ { j = 1 } ^ { D } \left(U\left(\alpha_{j t}, D_{j t}\right)+V^{\mathrm{s}}\left(L_{j}, D R_{j t}\right)\right.\right. \\
& \left.\left.-C\left(\theta_{j}, D R_{j t}\right)\right)-\sum_{g=1}^{G}\left(C_{g t}+S_{g t}\right)\right] \\
& \sum_{g=1}^{G} P_{g t}=\sum_{j=1}^{D}\left(D_{j t}^{\mathrm{o}}-D R_{j t}\right) \quad \forall g \in G, \forall t \in T \\
& -F_{l}^{\max } \leq B_{i j}\left(\theta_{i t}-\theta_{j t}\right) \leq F_{l}^{\max } \quad \forall l, \forall t \\
& D_{j t}^{\mathrm{a}}=\left(1-D R L_{j t}^{\max }\right) D_{j t}^{\mathrm{o}}+\sum_{k=1}^{M} D_{j k t}^{\mathrm{a}} \quad \forall j, \forall t \\
& \left(1-D R L_{j t}^{\max }\right) D_{j t}^{\mathrm{o}} \leq D_{j t}^{\mathrm{a}} \leq D_{j t}^{\mathrm{o}} \quad \forall j, \forall t \\
& 0 \leq D R_{j t} \leq D R L_{j t}^{\max } \cdot D_{j t}^{\mathrm{o}} \cdot I_{j t} \quad \forall j, \forall t \\
& D_{j t}^{\mathrm{a}}=D_{j t}^{\mathrm{o}}-D R_{j t} \quad \forall j, \forall t
\end{aligned}
$$

Equation (34) is subject to (4), (10), (11), (19), (20), (24)-(28), (31), (32) as well. The optimization goal (34) is to maximize social welfare using the compensation mechanism, where the first term represents the aggregate utilities of all customers, the second and the third terms represent the objective of the compensation mechanism (15), and the last two terms represent the unit commitment cost and the startup cost of thermal units. Equation (35) depicts the supply-demand balance requirement of system considering DR, associate the dual variable $\lambda_{t}$ with (35), which is also the MCP of the system. Equation (36) gives the network power flow limits on transmission lines, associate the dual variables $\pi_{l t}^{+}$and $\pi_{l t}^{-}$with (36). Equations (37) and (38) describe the actual load demand allowing for DR. Equation (39) gives the load curtailment capacity limits. Equation (40) calculates actual load demand. LMPs may be calculated as before using (33) after solving the model (4), (10), (11), (19), (20), (24)-(28), (31), (32), (34)-(40).

\section{Solution method}

Equation (4) is difficult to define explicitly in terms of load demand variables. To do this, it is first necessary to introduce a new set of binary variable $\sigma_{j t}$ to model the demand utility in an explicit manner [33, 34]. They satisfy:

$\sigma_{j t}= \begin{cases}1 & \bar{D}_{j}-D_{j t} \geq 0 \\ 0 & \text { otherwise }\end{cases}$

$\frac{\bar{D}_{j}-D_{j t}}{\sum_{g=1}^{G} P_{g}^{\max }} \leq \sigma_{j t} \leq 1+\frac{\bar{D}_{j}-D_{j t}}{\sum_{g=1}^{G} P_{g}^{\max }}$

From (41), if $\quad \bar{D}_{j}-D_{j t} \geq 0, \quad$ and considering $\bar{D}_{j} \leq \sum_{g=1}^{G} P_{g}^{\max }$, the lower bound of (42) must be strictly greater than zero and less than 1, while the upper bound is greater than 1 . Since $\sigma_{j t}$ is a binary variable, then if $\bar{D}_{j}-D_{j t} \geq 0$, it must be equal to 1 . A similar argument applies when $\bar{D}_{j}-D_{j t} \leq 0$ in which case $\sigma_{j t}=0$.

Analogously, the same method is applied to solve (19) and (20).

By these means, the demand utility function can be formulated as the sum of the products of some binary variables and a bounded continuous variable. After it is linearized, the proposed mathematical model is formulated as a MILP problem and it can be solved with the commercial MILP solver CPLEX.

\section{Case studies}

Numerical experiments are carried on a six-bus system and the modified IEEE 118-bus system to demonstrate the validity and effectiveness of the proposed model. In order to analyze the impacts of customer demand behavior on the MCP, LMPs, customer demand utility and social welfare, all these results are analyzed comparing the social welfare maximization model with a constant demand benefit function and with the customer utility function. All experiments are implemented on a personal computer with Inter (R) Core (TM) Duo 3.2 GHz CPU and 4 GB memory and programmed using Visual Studio $2010 \mathrm{C}++$, and the MILP solver is CPLEX 12.5. The simulated time interval is one day and is divided into 24 periods.

\subsection{Six-bus system}

A six-bus system with 3 thermal units, 3 load nodes, and 7 branches is shown in Fig. 3. All the parameters can be found in [35]. Three cases are used to illustrate the impacts of different factors on the dispatch results. 
Case 1: The NCUC model without DR, i.e. $D R L=0$.

Case 2: The integrated generation-consumption dispatch model considering the demand utility function with $D R L=0$, and compare the proposed model with the traditional one that has a fixed electric energy price.

Case 3: The DR compensation mechanism is integrated into the generation-consumption dispatch model, $D R L \neq 0$.

\subsubsection{Case 1 analysis}

The NCUC model is listed in (21)-(32) with the first term of (21) removed. Its aim is to obtain the maximum and the minimum LMPs of each load bus, and these are shown in Table 1.

We assume that the maximum and the minimum LMPs of each load bus are equal to $M U_{j t}^{\mathrm{H}}$ and $M U_{j t}^{\mathrm{o}}$, respectively. Hence, we deduce the customer's information through (3), (7) and (8). The results are shown in Table 2.

\subsubsection{Case 2 analysis}

\section{1) Dispatch results}

The dispatch results from the integrated method with $D R L=0$ are shown in Table 3. The customer's demand utility can be deduced through (4) after we obtain the customer's information $\alpha_{j t}$ and $\beta_{j t}$ from Case 1 as discussed above. The demand utility for each customer and in total are shown in Table 4.

In this case, the energy production cost is $132 \mathrm{k} \$$, the startup cost of units is $808 \mathrm{k} \$$, the total demand utility of customers is $2095 \mathrm{k} \$$, the social welfare is $19623 \mathrm{k} \$$.

\section{2) Comparison with traditional fixed-price strategy}

The proposed distributed real-time market clearing pricing strategy $\left(\lambda_{t}\right.$ in Section 3.1$)$ can be compared with

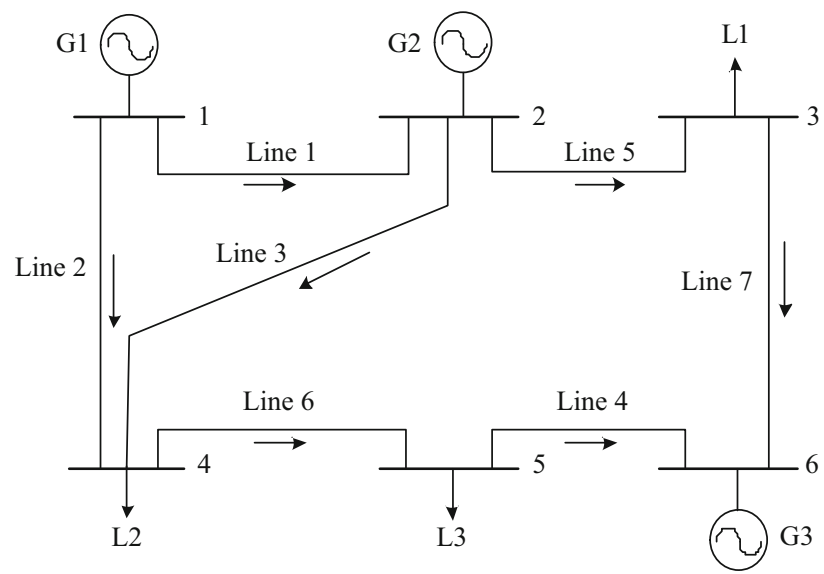

Fig. 3 Line diagram of six-bus system
Table 1 Demand LMPs for NCUC model

\begin{tabular}{|c|c|c|c|c|c|c|}
\hline \multirow[t]{2}{*}{ Period } & \multicolumn{6}{|c|}{ Demand LMP (\$/MWh) } \\
\hline & Bus 1 & Bus 2 & Bus 3 & Bus 4 & Bus 5 & Bus \\
\hline 1 & 17.03 & 17.03 & 17.03 & 17.03 & 17.03 & 17.03 \\
\hline 2 & 17.03 & 17.03 & 17.03 & 17.03 & 17.03 & 17.03 \\
\hline 3 & 17.03 & 17.03 & 17.03 & 17.03 & 17.03 & 17.03 \\
\hline 4 & 17.03 & 17.03 & 17.03 & 17.03 & 17.03 & 17.03 \\
\hline 5 & 17.03 & 17.03 & 17.03 & 17.03 & 17.03 & 17.03 \\
\hline 6 & 17.03 & 17.03 & 17.03 & 17.03 & 17.03 & 17.03 \\
\hline 7 & 17.03 & 40.69 & 43.05 & 55.51 & 53.14 & 44.20 \\
\hline 8 & 17.03 & 40.76 & 43.13 & 55.63 & 53.26 & 44.29 \\
\hline 9 & 17.03 & 40.69 & 43.05 & 55.51 & 53.14 & 44.20 \\
\hline 10 & 17.03 & 40.76 & 43.13 & 55.63 & 53.26 & 44.29 \\
\hline 11 & 17.03 & 40.83 & 43.21 & 55.75 & 53.37 & 44.37 \\
\hline 12 & 17.03 & 40.83 & 43.21 & 55.75 & 53.37 & 44.37 \\
\hline 13 & 17.03 & 40.83 & 43.21 & 55.75 & 53.37 & 44.37 \\
\hline 14 & 17.03 & 40.83 & 43.21 & 55.75 & 53.37 & 44.37 \\
\hline 15 & 17.03 & 79.39 & 85.63 & 118.50 & 112.20 & 88.66 \\
\hline 16 & 17.03 & 21.43 & 21.86 & 24.18 & 23.74 & 22.08 \\
\hline 17 & 17.03 & 21.46 & 21.90 & 24.24 & 23.80 & 22.12 \\
\hline 18 & 17.03 & 17.03 & 17.03 & 17.03 & 17.03 & 17.03 \\
\hline 19 & 17.03 & 21.50 & 21.94 & 24.30 & 23.85 & 22.16 \\
\hline 20 & 17.03 & 17.03 & 17.03 & 17.03 & 17.03 & 17.03 \\
\hline 21 & 17.03 & 17.03 & 17.03 & 17.03 & 17.03 & 17.03 \\
\hline 22 & 17.03 & 40.69 & 43.05 & 55.51 & 53.14 & 44.20 \\
\hline 23 & 17.03 & 40.69 & 43.05 & 55.51 & 53.14 & 44.20 \\
\hline 24 & 22.08 & 22.08 & 22.08 & 22.08 & 22.08 & 22.08 \\
\hline
\end{tabular}

Table 2 Estimated parameters of demand utility function at each load bus using NCUC results

\begin{tabular}{llll}
\hline Bus & $\alpha_{j t}$ & $\beta_{j t}$ & $\bar{D}_{j}(\mathrm{MW})$ \\
\hline Bus 3 & 359.99 & 1.827553 & 196.9792 \\
Bus 4 & 524.34 & 2.289513 & 229.0181 \\
Bus 5 & 492.84 & 4.831536 & 102.0048
\end{tabular}

the traditional fixed-price strategy [36]. The NCUC program has been implemented using a MILP algorithm to obtain the initial price in a centralized manner. Given the initial price at each period of time $t \in T$, customers of each type $\alpha$ determine their optimal demand. The type $\alpha^{\max }$ has the most demand, and this paper assumes that all of the customers are of type $\alpha^{\max }$, representing the worst case.

If a customer's demand is $D_{j t}(\mathrm{MW})$ and the price is $\lambda_{t}$ (\$/MWh) during time interval $t \in T$, the welfare of each customer can be simply expressed as:

$W\left(\alpha_{j t}, D_{j t}\right)=U\left(\alpha_{j t}, D_{j t}\right)-\lambda_{t} D_{j t}$ 
Table 3 Results of unit commitment and generation dispatch with $D L R=0$

\begin{tabular}{|c|c|c|c|c|c|c|}
\hline \multirow[t]{2}{*}{ Period } & \multicolumn{3}{|c|}{ UC status } & \multicolumn{3}{|c|}{ Generation schedule (MW) } \\
\hline & G1 & G2 & G3 & G1 & G2 & G3 \\
\hline 1 & 1 & 0 & 0 & 203.4 & 0 & 0 \\
\hline 2 & 1 & 0 & 0 & 218.4 & 0 & 0 \\
\hline 3 & 1 & 0 & 0 & 217.8 & 0 & 0 \\
\hline 4 & 1 & 0 & 0 & 219.7 & 0 & 0 \\
\hline 5 & 1 & 0 & 1 & 211.8 & 0 & 10.00 \\
\hline 6 & 1 & 0 & 1 & 216.9 & 0 & 10.00 \\
\hline 7 & 1 & 1 & 1 & 212.7 & 20.98 & 20.00 \\
\hline 8 & 1 & 1 & 1 & 206.2 & 43.28 & 20.00 \\
\hline 9 & 1 & 1 & 1 & 208.8 & 34.31 & 20.00 \\
\hline 10 & 1 & 1 & 0 & 206.8 & 54.19 & 0 \\
\hline 11 & 1 & 1 & 1 & 191.6 & 93.37 & 20.00 \\
\hline 12 & 1 & 1 & 1 & 191.8 & 92.70 & 20.00 \\
\hline 13 & 1 & 1 & 1 & 192.5 & 90.19 & 20.00 \\
\hline 14 & 1 & 1 & 1 & 193.5 & 86.80 & 20.00 \\
\hline 15 & 1 & 1 & 1 & 192.2 & 91.08 & 20.00 \\
\hline 16 & 1 & 1 & 1 & 205.8 & 51.08 & 10.23 \\
\hline 17 & 1 & 1 & 1 & 216.9 & 11.08 & 13.34 \\
\hline 18 & 1 & 0 & 1 & 219.0 & 0 & 10.00 \\
\hline 19 & 1 & 0 & 1 & 219.2 & 0 & 18.39 \\
\hline 20 & 1 & 0 & 1 & 210.3 & 0 & 10.00 \\
\hline 21 & 1 & 0 & 1 & 215.8 & 0 & 10.00 \\
\hline 22 & 1 & 1 & 1 & 209.0 & 33.76 & 20.00 \\
\hline 23 & 1 & 1 & 1 & 209.0 & 33.65 & 20.00 \\
\hline 24 & 1 & 0 & 1 & 220.0 & 0 & 10.84 \\
\hline
\end{tabular}

where $W\left(\alpha_{j t}, D_{j t}\right)$ is customer's welfare function; $\lambda_{t} D_{j t}$ is customer's electricity cost. For each price $\lambda_{t}$, customers try to adjust their electricity consumption $D_{j t}$ to maximize their own welfare $W\left(\alpha_{j t}, D_{j t}\right)$, and the optimal consumption is achieved when the first derivative of (43) equals zero. Additionally, the customer's marginal utility is equal to the market price. Hence, the fixed price $\lambda_{t}^{\text {fixed }}$ in each period $t \in T$ is calculated as:

$\lambda_{t}^{\text {fixed }}=\alpha_{j t}^{\max }-\frac{\beta_{j t} \sum_{g=1}^{G} P_{g t} u_{g t}}{D}$

The calculated average welfare for the proposed distributed real-time pricing and for fixed pricing are compared in Fig. 4. It should be noted that the average welfare of each customer using the proposed distributed real-time pricing algorithm is much higher than that for the traditional fixed pricing algorithm. This demonstrates that distributed real-time pricing is an effective tool because the price interacts with customer behavior.
Table 4 Demand utility of all customers with $D L R=0$

\begin{tabular}{|c|c|c|c|c|}
\hline \multirow[t]{2}{*}{ Period } & \multicolumn{3}{|c|}{ Demand utility (k\$) } & \multirow[t]{2}{*}{ Total utility $(\mathrm{k} \$)$} \\
\hline & Bus 3 & Bus 4 & Bus 5 & \\
\hline 1 & 21.90 & 37.53 & 15.68 & 75.11 \\
\hline 2 & 23.11 & 39.58 & 16.54 & 79.23 \\
\hline 3 & 23.06 & 39.49 & 16.51 & 79.06 \\
\hline 4 & 23.21 & 39.75 & 16.61 & 79.57 \\
\hline 5 & 23.37 & 40.04 & 16.73 & 80.14 \\
\hline 6 & 23.76 & 40.69 & 17.01 & 81.47 \\
\hline 7 & 25.72 & 44.02 & 18.40 & 88.15 \\
\hline 8 & 26.79 & 45.83 & 19.16 & 91.79 \\
\hline 9 & 26.37 & 45.12 & 18.86 & 90.35 \\
\hline 10 & 26.22 & 44.87 & 18.76 & 89.84 \\
\hline 11 & 28.97 & 49.51 & 20.70 & 99.17 \\
\hline 12 & 28.94 & 49.46 & 20.68 & 99.08 \\
\hline 13 & 28.84 & 49.29 & 20.61 & 98.74 \\
\hline 14 & 28.70 & 49.06 & 20.51 & 98.27 \\
\hline 15 & 28.87 & 49.35 & 20.63 & 98.86 \\
\hline 16 & 26.63 & 45.56 & 19.05 & 91.24 \\
\hline 17 & 24.84 & 42.52 & 17.77 & 85.13 \\
\hline 18 & 23.92 & 40.97 & 17.12 & 82.01 \\
\hline 19 & 24.56 & 42.05 & 17.58 & 84.20 \\
\hline 20 & 23.25 & 39.83 & 16.65 & 79.72 \\
\hline 21 & 23.68 & 40.55 & 16.95 & 81.18 \\
\hline 22 & 26.34 & 45.07 & 18.84 & 90.26 \\
\hline 23 & 26.34 & 45.06 & 18.84 & 90.24 \\
\hline 24 & 24.06 & 41.21 & 17.22 & 82.49 \\
\hline Total & 611.45 & 1046.41 & 437.43 & 2095.29 \\
\hline
\end{tabular}

The computation efficiency of the two methods is considered in Table 5, where it can be seen that the total computational time and the number of iterations for distributed real-time pricing strategy are significantly reduced compared with fixed pricing strategy.

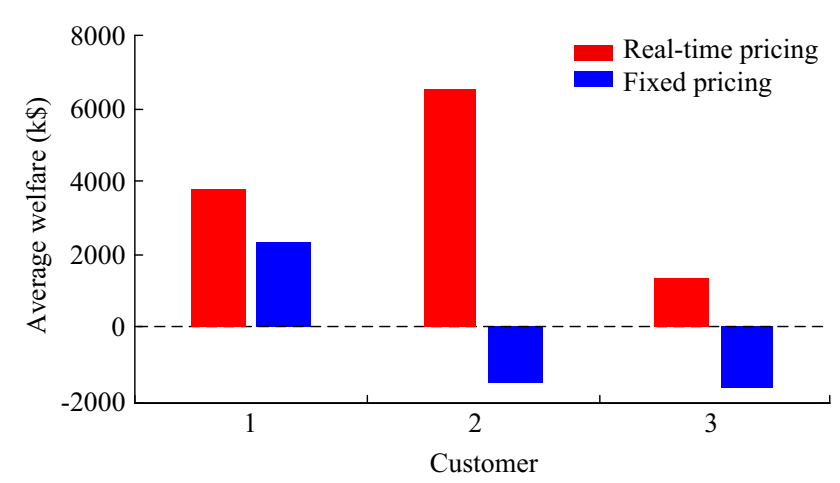

Fig. 4 Average customer welfare considering demand utility and with fixed price 
Table 5 Computation efficiency of two methods

\begin{tabular}{llc}
\hline Method & Time $(\mathrm{s})$ & Iteration \\
\hline Real-time pricing & 0.194 & 7 \\
Fixed pricing & 0.236 & 11 \\
\hline
\end{tabular}

Table 6 Customer attributes

\begin{tabular}{|c|c|c|c|c|c|c|}
\hline \multirow[t]{2}{*}{ Period } & \multicolumn{3}{|c|}{ Locational value $L_{j}$} & \multicolumn{3}{|c|}{ Preference parameter $\theta_{j}$} \\
\hline & Bus 3 & Bus 4 & Bus 5 & Bus 3 & Bus 4 & Bus 5 \\
\hline 1 & 0.3 & 0.4 & 0.4 & 0.2 & 0.4 & 0.3 \\
\hline 2 & 0.4 & 0.3 & 0.3 & 0.1 & 0.3 & 0.2 \\
\hline 3 & 0.3 & 0.4 & 0.3 & 0.1 & 0.3 & 0.2 \\
\hline 4 & 0.4 & 0.5 & 0.4 & 0.2 & 0.4 & 0.3 \\
\hline 5 & 0.4 & 0.4 & 0.4 & 0.3 & 0.5 & 0.4 \\
\hline 6 & 0.5 & 0.5 & 0.4 & 0.4 & 0.6 & 0.5 \\
\hline 7 & 0.7 & 0.8 & 0.7 & 0.7 & 0.7 & 0.7 \\
\hline 8 & 0.8 & 0.8 & 0.8 & 0.7 & 0.7 & 0.6 \\
\hline 9 & 0.7 & 0.8 & 0.8 & 0.7 & 0.7 & 0.6 \\
\hline 10 & 0.8 & 0.7 & 0.8 & 0.6 & 0.8 & 0.7 \\
\hline 11 & 0.9 & 0.8 & 0.9 & 0.6 & 0.8 & 0.7 \\
\hline 12 & 0.9 & 0.8 & 0.8 & 0.7 & 0.9 & 0.8 \\
\hline 13 & 0.9 & 0.8 & 0.8 & 0.7 & 0.9 & 0.8 \\
\hline 14 & 0.9 & 0.9 & 0.9 & 0.8 & 0.8 & 0.8 \\
\hline 15 & 0.9 & 0.9 & 0.9 & 0.9 & 0.9 & 0.9 \\
\hline 16 & 0.6 & 0.7 & 0.6 & 0.8 & 0.7 & 0.6 \\
\hline 17 & 0.6 & 0.6 & 0.5 & 0.5 & 0.5 & 0.6 \\
\hline 18 & 0.3 & 0.4 & 0.4 & 0.5 & 0.5 & 0.5 \\
\hline 19 & 0.6 & 0.8 & 0.7 & 0.4 & 0.6 & 0.5 \\
\hline 20 & 0.6 & 0.5 & 0.4 & 0.5 & 0.7 & 0.6 \\
\hline 21 & 0.4 & 0.6 & 0.5 & 0.6 & 0.7 & 0.7 \\
\hline 22 & 0.7 & 0.6 & 0.8 & 0.8 & 0.8 & 0.8 \\
\hline 23 & 0.8 & 0.7 & 0.7 & 0.9 & 0.9 & 0.9 \\
\hline 24 & 0.6 & 0.5 & 0.6 & 0.4 & 0.6 & 0.5 \\
\hline
\end{tabular}

\subsubsection{Case 3 analysis}

1) DR compensation mechanism

As mentioned in Section 2.2, the locational value and the preference parameter of customers are the two critical parameters of the DR compensation mechanism, and the locational value can be calculated according to literature [19]. The calculated locational values and assumed preference parameters are listed in Table 6.

To demonstrate the advantage and validity of the proposed compensation mechanism, we take customer 3 of Bus 3 in period 12 as an example. Its locational value is $L_{3}=0.9$, and its true preference parameter is $\theta_{3}=0.7$. According to (19) and (20), the optimal DR amount and compensation fees are obtained, and they are graphed in Fig. 5 .

Customers may lie about their private information $\theta_{3}$ to receive more monetary compensation $y$ offered by energy supplier. The proposed compensation mechanism avoids this because only customers who report their true private information can obtain maximum utility (18). According to (13), we can obtain customers' benefit, and Fig. 6 shows the benefit of customer 3 for all possible private information $\theta_{3}$. The benefit of customer 3 is maximized when he or she reports the truth at $\theta_{3}=0.7$. Hence, each customer better reports his or her true private information $\theta_{3}$ and thereby selects a compensation scheme according to their true willingness to provide DR.

2) Dispatch results

The consumers respond to the market energy price based on their voluntary psychological feelings and then determine whether and how much to curtail. The optimal DR amounts of customers can be solved by co-optimizing DR and generation-consumption dispatch decisions

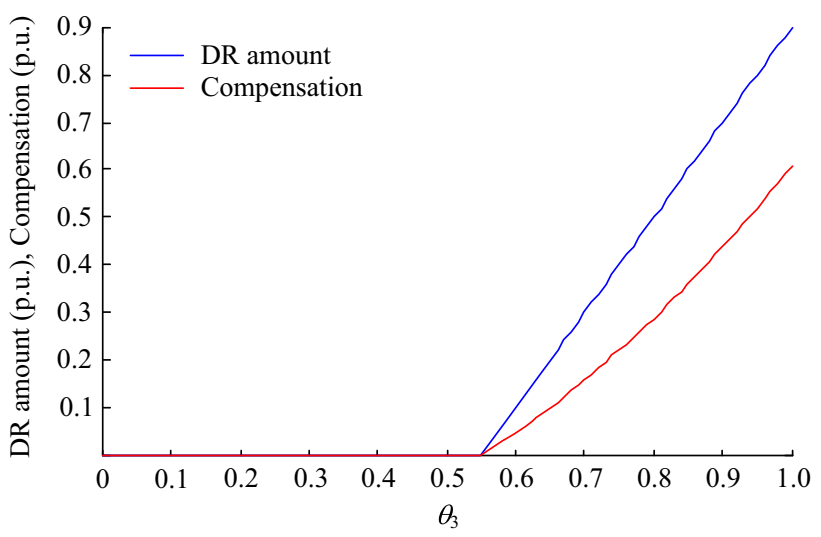

Fig. 5 DR amount and compensation fees of proposed compensation mechanism for customer 3 on Bus 3

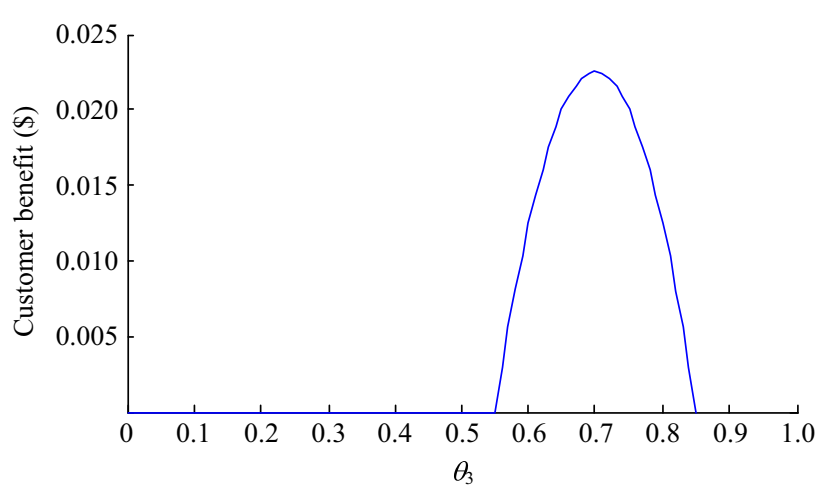

Fig. 6 Customer benefit for $L_{3}=0.9$ and $\theta_{3}=0.7$ 
Table 7 Optimal DR amounts for three types of customers in six-bus system

\begin{tabular}{|c|c|c|c|c|c|c|c|}
\hline \multirow[t]{2}{*}{ Period } & \multicolumn{3}{|c|}{ DR amount (MW) } & \multirow[t]{2}{*}{ Period } & \multicolumn{3}{|c|}{ DR amount (MW) } \\
\hline & Bus 3 & Bus 4 & Bus 5 & & Bus 3 & Bus 4 & Bus 5 \\
\hline 1 & 0 & 0 & 0 & 14 & 5 & 5 & 5 \\
\hline 2 & 0 & 0 & 0 & 15 & 7 & 7 & 7 \\
\hline 3 & 0 & 0 & 0 & 16 & 2 & 1 & 0 \\
\hline 4 & 0 & 0 & 0 & 17 & 0 & 0 & 0 \\
\hline 5 & 0 & 0 & 0 & 18 & 0 & 0 & 0 \\
\hline 6 & 0 & 0 & 0 & 19 & 0 & 0 & 0 \\
\hline 7 & 1 & 2 & 1 & 20 & 0 & 0 & 0 \\
\hline 8 & 2 & 2 & 0 & 21 & 0 & 0 & 0 \\
\hline 9 & 1 & 2 & 0 & 22 & 3 & 2 & 4 \\
\hline 10 & 0 & 3 & 2 & 23 & 6 & 5 & 5 \\
\hline 11 & 1 & 4 & 3 & 24 & 0 & 0 & 0 \\
\hline 12 & 3 & 6 & 4 & Total & 34 & 45 & 35 \\
\hline 13 & 3 & 6 & 4 & & & & \\
\hline
\end{tabular}

Table 8 Components of DR compensation of customers

\begin{tabular}{|c|c|c|c|c|c|}
\hline Period & DR (MW) & $\mathrm{CC}(\$)$ & OC (\$) & CR (\$) & SR $(\$)$ \\
\hline 1 & 0 & 0 & 0 & 0 & 0 \\
\hline 2 & 0 & 0 & 0 & 0 & 0 \\
\hline 3 & 0 & 0 & 0 & 0 & 0 \\
\hline 4 & 0 & 0 & 0 & 0 & 0 \\
\hline 5 & 0 & 0 & 0 & 0 & 0 \\
\hline 6 & 0 & 0 & 0 & 0 & 0 \\
\hline 7 & 4 & 1650 & 1500 & 150 & 1350 \\
\hline 8 & 4 & 1800 & 1600 & 200 & 1400 \\
\hline 9 & 3 & 1275 & 1150 & 125 & 1025 \\
\hline 10 & 5 & 2175 & 1850 & 325 & 1525 \\
\hline 11 & 8 & 4050 & 3400 & 650 & 2750 \\
\hline 12 & 13 & 6875 & 5350 & 1525 & 3825 \\
\hline 13 & 13 & 6875 & 5350 & 1525 & 3825 \\
\hline 14 & 15 & 8625 & 6750 & 1875 & 4875 \\
\hline 15 & 21 & 13125 & 9450 & 3675 & 5775 \\
\hline 16 & 3 & 1075 & 950 & 125 & 825 \\
\hline 17 & 0 & 0 & 0 & 0 & 0 \\
\hline 18 & 0 & 0 & 0 & 0 & 0 \\
\hline 19 & 0 & 0 & 0 & 0 & 0 \\
\hline 20 & 0 & 0 & 0 & 0 & 0 \\
\hline 21 & 0 & 0 & 0 & 0 & 0 \\
\hline 22 & 9 & 3975 & 3250 & 725 & 2525 \\
\hline 23 & 16 & 8050 & 5900 & 2150 & 3750 \\
\hline 24 & 0 & 0 & 0 & 0 & 0 \\
\hline Total & 114 & 59550 & 46500 & 13050 & 33450 \\
\hline
\end{tabular}

simultaneously. According to the dispatch model in Section 3.2, the optimal DR amounts are shown in Table 7.
Using the data in Table 7, the $D R L=1.88 \%$ according to (5). Different types of customers have different DR preferences in different periods.

Meanwhile, the optimal DR compensation mechanism for consumers can be obtained. The curtailing capacity (i.e. $\mathrm{DR})$, the compensation cost (CC), the outage cost (OC), the consumer revenue (CR), and the energy supply side revenue (SR) are shown in Table 8. The demand utility for each customer and in total are shown in Table 9. The LMPs of each bus are shown in Table 10.

In this case, the energy production cost is $122 \mathrm{k} \$$, the startup cost of units is $808 \mathrm{k} \$$, the total demand utility of customers is $2070 \mathrm{k} \$$, the revenue generated by the DR compensation mechanism is $47 \mathrm{k} \$$, and the social welfare is $1994 \mathrm{k} \$$.

In addition, in order to emphasize the importance of the two critical parameters $\left(L_{j}, \theta_{j}\right)$, some further tests are performed. On one hand, $L_{j}$ is fixed at 0.7 for all the customers during study cycle, as if all the customers were at the same location. On the other hand, $\theta_{j}$ is fixed at 0.7 for all the customers during study cycle, so all customers have the same preference. Table 11 compares these results.

With fixed $L_{j}=0.7$ or fixed $\theta_{j}=0.7$, the total demand utility of all customers is increased but the amount of DR, $\mathrm{CC}, \mathrm{OC}, \mathrm{CR}$ and $\mathrm{SR}$, as well as the social welfare are reduced. Therefore, when $L_{j}$ and $\theta_{j}$ are fixed to a certain value, the dispatch results are non-optimal. Only customers who share their true information, and select a compensation scheme according to their true willingness to provide DR, can achieve maximum benefit. Therefore, the proposed compensation mechanism benefits both the suppliers and their customers, and realize maximum social welfare and Pareto optimal results.

The computing time is also listed in Table 11, demonstrating the applicability of our model to the six-bus system.

\section{3) Comparison of Case 3 with Case 2}

The system demand, the LMP, the customers' demand utility and the social welfare with and without DR are shown in Fig. 7a to Fig. 7d, respectively.

Figure 7a compares the system demand without DR $(D R L=0)$ and the expected system demand with $D R L=1.88 \%$ as determined above from optimal decisionmaking results. It illustrates that the compensation mechanism encourages customers to curtail a certain amount of load.

Figure $7 \mathrm{~b}$ shows the LMP with $D R L=0$ and with $D R L=1.88 \%$. It can be seen that the LMP decreases due to the demand curtailment of customers participating in DR during peak periods. However, the LMP of Bus 4 is increased to $55.55 \$ / \mathrm{MWh}$ as compared with that of Case 
Table 9 Demand utility with optimal DR

\begin{tabular}{|c|c|c|c|c|}
\hline \multirow[t]{2}{*}{ Period } & \multicolumn{3}{|c|}{ Demand utility (k\$) } & \multirow{2}{*}{ Total utility $(\mathrm{k} \$)$} \\
\hline & Bus 3 & Bus 4 & Bus 5 & \\
\hline 1 & 21.90 & 37.53 & 15.68 & 75.11 \\
\hline 2 & 23.11 & 39.58 & 16.54 & 79.23 \\
\hline 3 & 23.06 & 39.49 & 16.51 & 79.06 \\
\hline 4 & 23.21 & 39.75 & 16.61 & 79.57 \\
\hline 5 & 23.37 & 40.04 & 16.73 & 80.14 \\
\hline 6 & 23.76 & 40.69 & 17.01 & 81.47 \\
\hline 7 & 25.53 & 43.48 & 18.15 & 87.15 \\
\hline 8 & 26.43 & 45.32 & 19.16 & 90.91 \\
\hline 9 & 26.19 & 44.59 & 18.86 & 89.64 \\
\hline 10 & 26.22 & 44.07 & 18.25 & 88.54 \\
\hline 11 & 28.81 & 48.61 & 20.06 & 97.48 \\
\hline 12 & 28.47 & 48.10 & 19.81 & 96.38 \\
\hline 13 & 28.36 & 47.92 & 19.73 & 96.01 \\
\hline 14 & 27.89 & 47.91 & 19.39 & 95.19 \\
\hline 15 & 27.74 & 47.75 & 19.06 & 94.54 \\
\hline 16 & 26.27 & 45.30 & 19.05 & 90.62 \\
\hline 17 & 24.84 & 42.52 & 17.77 & 85.13 \\
\hline 18 & 23.92 & 40.97 & 17.12 & 82.01 \\
\hline 19 & 24.56 & 42.05 & 17.58 & 84.20 \\
\hline 20 & 23.25 & 39.83 & 16.65 & 79.72 \\
\hline 21 & 23.68 & 40.55 & 16.95 & 81.18 \\
\hline 22 & 25.79 & 44.55 & 17.82 & 88.15 \\
\hline 23 & 25.21 & 43.73 & 17.55 & 86.48 \\
\hline 24 & 24.06 & 41.21 & 17.22 & 82.49 \\
\hline Total & 605.63 & 1035.51 & 429.27 & 2070.40 \\
\hline
\end{tabular}

2, illustrating that, when deploying the DR compensation mechanism with the objective of maximizing the social welfare, LMPs at some buses may increase. In this case, the higher LMP is primarily induced by congestion on Line 2 . Dynamic unit commitment is affected by the units' start-up costs, which changes the choice of marginal unit. Thus increasing social welfare does not necessarily reduce LMP.

Figure 7c shows the total demand utility of customers with $D R L=0$ and with $D R L=1.88 \%$. When the units' output can meet the original demand of customers, the demand utility of customers is at a maximum. Therefore, the demand utility of customers is reduced by the demand curtailment of customers participating in DR.

Figure $7 \mathrm{~d}$ shows the social welfare with $D R L=0$ and with $D R L=1.88 \%$. The social welfare increases very significantly with $D R L=1.88 \%$ because the customers' utility loss from DR is compensated by the cost savings of the generation side. The proposed compensation mechanism can increase the benefits both the supply-side and demand-side. The main reason is that the reduction in demand utility of customers is approximately $1.2 \%$, from
Table 10 LMP of each bus with optimal DR

\begin{tabular}{|c|c|c|c|c|c|c|}
\hline \multirow[t]{2}{*}{ Period } & \multicolumn{6}{|c|}{ LMP (\$/MWh) } \\
\hline & Bus 1 & Bus 2 & Bus 3 & Bus 4 & Bus 5 & Bus $\theta$ \\
\hline 1 & 17.03 & 17.03 & 17.03 & 17.03 & 17.03 & 17.03 \\
\hline 2 & 17.03 & 17.03 & 17.03 & 17.03 & 17.03 & 17.03 \\
\hline 3 & 17.03 & 17.03 & 17.03 & 17.03 & 17.03 & 17.03 \\
\hline 4 & 17.03 & 17.03 & 17.03 & 17.03 & 17.03 & 17.03 \\
\hline 5 & 17.03 & 17.03 & 17.03 & 17.03 & 17.03 & 17.03 \\
\hline 6 & 17.03 & 17.03 & 17.03 & 17.03 & 17.03 & 17.03 \\
\hline 7 & 17.03 & 40.69 & 43.02 & 55.46 & 53.10 & 44.17 \\
\hline 8 & 17.03 & 40.76 & 43.08 & 55.56 & 53.19 & 44.23 \\
\hline 9 & 17.03 & 40.69 & 43.06 & 55.52 & 53.16 & 44.21 \\
\hline 10 & 17.03 & 40.76 & 43.11 & 55.59 & 53.22 & 44.26 \\
\hline 11 & 17.03 & 40.83 & 43.20 & 55.74 & 53.36 & 44.36 \\
\hline 12 & 17.03 & 40.83 & 43.18 & 55.71 & 53.33 & 44.34 \\
\hline 13 & 17.03 & 40.83 & 43.18 & 55.70 & 53.32 & 44.33 \\
\hline 14 & 17.03 & 40.76 & 43.16 & 55.67 & 53.30 & 44.32 \\
\hline 15 & 17.03 & 40.76 & 43.15 & 55.65 & 53.28 & 44.30 \\
\hline 16 & 17.03 & 40.76 & 43.08 & 55.55 & 53.18 & 44.23 \\
\hline 17 & 17.03 & 21.46 & 21.90 & 24.22 & 23.78 & 22.12 \\
\hline 18 & 17.03 & 17.03 & 17.03 & 17.03 & 17.03 & 17.03 \\
\hline 19 & 17.03 & 21.50 & 21.95 & 24.29 & 23.84 & 22.16 \\
\hline 20 & 17.03 & 17.03 & 17.03 & 17.03 & 17.03 & 17.03 \\
\hline 21 & 17.03 & 17.03 & 17.03 & 17.03 & 17.03 & 17.03 \\
\hline 22 & 17.03 & 40.68 & 43.04 & 55.48 & 53.12 & 44.19 \\
\hline 23 & 17.03 & 40.65 & 43.01 & 55.44 & 53.08 & 44.16 \\
\hline 24 & 22.08 & 22.08 & 22.08 & 22.08 & 22.08 & 22.08 \\
\hline
\end{tabular}

$2095 \mathrm{k} \$$ to $2070 \mathrm{k} \$$, while the total cost saving for generators is approximately $7.4 \%$, from $132 \mathrm{k} \$$ to $122 \mathrm{k} \$$, due to not starting up the unit with higher marginal cost.

\subsection{IEEE 118-bus system}

A modified IEEE 118-bus test system is used to test the proposed dispatch model at a more practical scale [37]. The system has 53 units, 186 branches, 14 capacitors, 9 tapchangers, and 91 load buses. The detailed IEEE 118-bus system data are given in motor.ece.iit.edu/data/SCUC_118. The dispatch results including DR, CC, OC, CR, and SR, and the computation time are shown in Table 12. The comments applying to Table 11 above apply equally to Table 12.

The system demand, LMP, demand utility and social welfare with and without DR are shown in Fig. 8a to Fig. 8d, respectively, as they were shown for the six-bus system in Fig. 7.

Figure 8a compares the system demand with $D R L=0$ and with $D R L=4.56 \%$ at bus 38 , as determined from the optimal decision-making results. A large amount of load is curtailed in the peak periods $10-16$ and $18-22$. 
Table 11 Comparison of optimal DR compensation mechanism with fixed $L_{j}=0.7$ and $\theta_{j}=0.7$ in six-bus system

\begin{tabular}{lcllllccc}
\hline Scenario & DR $(\mathrm{MW})$ & CC $(\mathrm{k} \$)$ & OC $(\mathrm{k} \$)$ & CR $(\mathrm{k} \$)$ & SR $(\mathrm{k} \$)$ & Demand utility $(\mathrm{k} \$)$ & Social welfare $(\mathrm{k} \$)$ & Time $(\mathrm{s})$ \\
\hline Optimal & 114 & 59.55 & 46.50 & 13.05 & 33.45 & 2.07 & 1993.73 & 0.077 \\
$L_{j}=0.7$ & 88 & 38.65 & 30.80 & 7.85 & 22.95 & 2095.29 & 1947.36 \\
$\theta_{j}=0.7$ & 80 & 33.15 & 24.90 & 8.73 & 25.05 & 2093.81 & 1951.07 & 0.088 \\
\hline
\end{tabular}

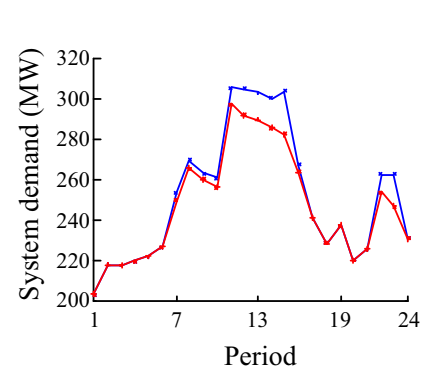

(a) System demand

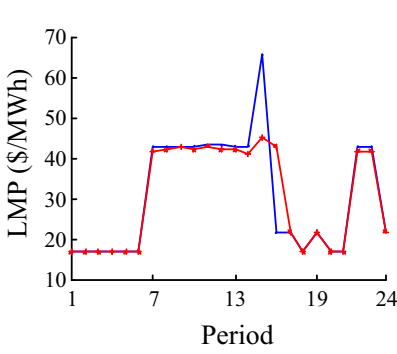

(b) LMP at Bus 4

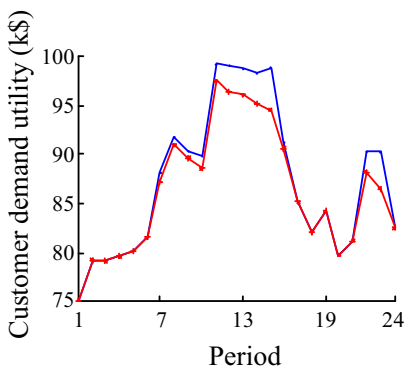

(c) Customer utility

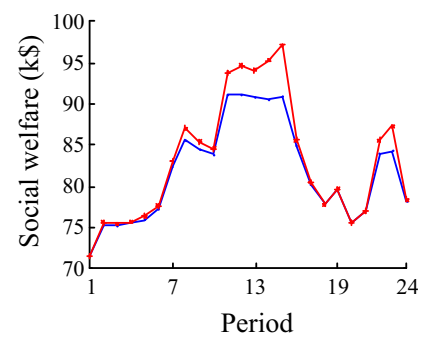

(d) Social welfare

$$
-D R L=0 ;-D R L=1.88 \%
$$

Fig. 7 System demand, LMP, customer demand utility and social welfare with and without DR for six-bus system

Table 12 Comparison of optimal DR compensation mechanism with fixed $L_{j}=0.7$ and $\theta_{j}=0.7$ in IEEE 118 -bus system

\begin{tabular}{llrrlllll}
\hline Scenario & DR $(\mathrm{MW})$ & CC $(\mathrm{k} \$)$ & OC $(\mathrm{k} \$)$ & CR $(\mathrm{k} \$)$ & SR $(\mathrm{k} \$)$ & Demand utility $(\mathrm{k} \$)$ & Social welfare $(\mathrm{k} \$)$ & Time $(\mathrm{s})$ \\
\hline Optimal & 338.9 & 1696.88 & 1338.35 & 358.53 & 979.83 & 1670.20 & 829.04 & 0.445 \\
$L_{j}=0.7$ & 325.1 & 1414.48 & 1134.35 & 280.13 & 854.23 & 1679.24 & 810.47 & 0.243 \\
$\theta_{j}=0.7$ & 195.2 & 919.95 & 698.70 & 233.58 & 697.05 & 1666.38 & 800.68 & 0.223 \\
\hline
\end{tabular}

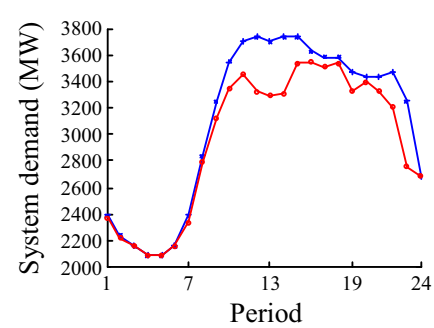

(a) System demand

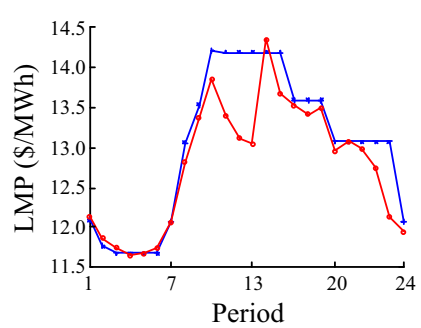

(b) LMP at bus 38

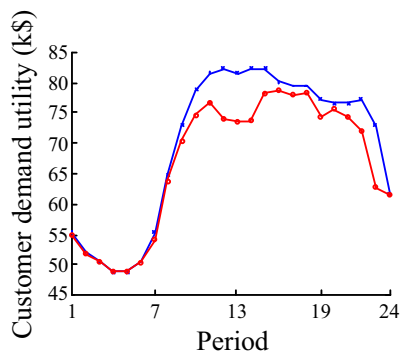

(c) Customer utility

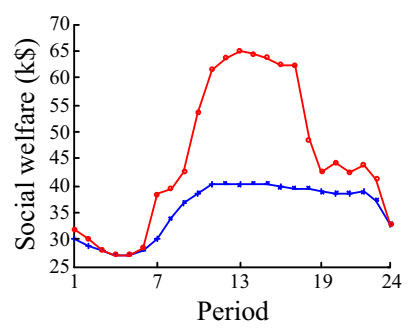

(d) Social welfare

$\rightarrow D R L=0 ;-D R L=4.56 \%$

Fig. 8 System demand, LMP, customer demand utility and social welfare with and without DR for IEEE118-bus system

Figure $8 \mathrm{~b}$ shows the LMP with $D R L=0$ and with $D R L=4.56 \%$ at bus 38 . Load curtailment occurs at peak periods, however, the LMP of bus 54 is increased to 14.42 $\$ / \mathrm{MWh}$ as compared with $14.13 \$ / \mathrm{MWh}$ for $D R L=0$, even the system load levels are smaller than those of the case $D R L=0$. The higher LMP is primarily induced by the congestion of line 54. Dynamic unit commitment is affected by the units' start-up cost, and the load curtailment at some buses may trigger new transmission congestions and change the marginal unit.

Figure $8 \mathrm{c}$ shows the demand utility of customers with $D R L=0$ and with $D R L=4.56 \%$. Figure $8 \mathrm{~d}$ shows the social welfare with $D R L=0$ and the social welfare with $D R L=4.56 \%$. The reduction in demand utility of 
customers is approximately $4.1 \%$, from $1670 \mathrm{k} \$$ to 1602 $\mathrm{k} \$$, a larger relative change compared with the six-bus system, while the total cost savings of generators is approximately $9 \%$ from $817 \mathrm{k} \$$ to $739 \mathrm{k} \$$.

\section{Conclusion}

Based on consumer behavior theory in microeconomics, this paper, from a novel perspective, establishes an integrated generation-consumption dispatch model considering the demand utility function. Additionally, the decisionmaking process of the DR compensation is incorporated into this dispatch model. The individual behavior of customers is seen to impact market equilibrium. Simulation results using a six-bus system and the modified IEEE 118-bus system verify the impact of behavior changes of customers on generation dispatch, social welfare, market clearing, LMP and the demand utility. All these results are analyzed by comparison with and without DR. Finally, compared with the traditional fixed pricing, the proposed method can increase the benefits to both the supply and demand sides, and the original demand utility of customers is not lost because it is compensated by participating in DR. Therefore, the Pareto optimality of system dispatch is achieved and social welfare maximization is realized as well.

Acknowledgement This work was supported by National Natural Science Foundation of China (No. 51477091, No. 51407106).

Open Access This article is distributed under the terms of the Creative Commons Attribution 4.0 International License (http:// creativecommons.org/licenses/by/4.0/), which permits unrestricted use, distribution, and reproduction in any medium, provided you give appropriate credit to the original author(s) and the source, provide a link to the Creative Commons license, and indicate if changes were made.

\section{Appendix A}

We present a simple example to illustrate the derivation process of the assumed values of $K_{1}$ and $K_{2}$. The energy supplier provides the different compensation fees for different types of customers. If a customer curtails $x(\mathrm{~kW})$ power energy at a rate of $\pi(\$ / \mathrm{kWh})$, he or she will obtain $\pi x$ dollars per hour of monetary compensation. Hence, the benefit function of the customer is:

$U^{\mathrm{d}}(\theta, x)=\pi x-c(\theta, x)$

Customers are rational, they will select to curtail $x$ of load to maximize their benefit, where $x$ satisfies the first order condition of its benefit function: $\frac{\partial U^{\mathrm{d}}}{\partial x}=\pi-\frac{\partial c}{\partial x}=0$

The compensation fees provided by the energy suppliers are not classified in detail. They only show the amount of power that each participating customer is willing to curtail, and the fee that the energy supplier is willing to pay them per $\mathrm{kWh}$. The main focus is to research energy consumption behaviors of customers.

An example will illustrate the basis for obtaining the coefficients $K_{1}$ and $K_{2}$ of the assumed outage cost function. According to the first order condition (A2), we have

$\pi-2 K_{1} x-K_{2}+K_{2} \theta=0$

Assume that there are $n$ customers participating in the demand response program. According to (A3), we know that $n$ customers need $n$ equations, there are $n+2$ unknown variables $\left(K_{1}, K_{2}\right.$ and all the $\left.\theta\right)$. Since $\theta$ is normalized as $0 \leq \theta \leq 1$, it sorts the customers from the least willing $(\theta=0)$ to the most willing $(\theta=1)$ to curtail load. Hence, there are $n$ equations and $n$ unknown variables. The compensation fee $\pi$ provided by the energy suppliers and the amount of curtailment $x$ for each customer is known. Therefore, this method can estimate the assumed values of $K_{1}$ and $K_{2}$, as well as the customer types $\theta$.

In order to illustrate the calibration method mentioned above, take 10 customers for example, and if a customer willing to curtail less load than $500 \mathrm{~kW}$, he or she will get paid $3.25 \$ / \mathrm{kWh}$. Otherwise, a customer willing to curtail more load than $500 \mathrm{~kW}$ will get paid $3 \$ / \mathrm{kWh}$. The amounts of load curtailment for each customer are shown in Table A1. The assumed values of $K_{1}, K_{2}$ and estimated values of all the customer types $\theta$ can be deduced using the described method, and are shown in Table A2 for this example.
Table A1 Load curtailment amount of each customer

\begin{tabular}{lc}
\hline Customer & Amount of load $(\mathrm{kW})$ \\
\hline 1 & 59 \\
2 & 100 \\
3 & 130 \\
4 & 134 \\
5 & 151 \\
6 & 184 \\
7 & 200 \\
8 & 349 \\
9 & 364 \\
10 & 770 \\
\hline
\end{tabular}


Table A2 Assumed coefficients and estimated types of 10 example customers

\begin{tabular}{llllll}
\hline$K_{1}$ & $K_{2}$ & $\theta_{1}$ & $\theta_{2}$ & $\theta_{3}$ & $\theta_{4}$ \\
\hline 0.0019 & 3.0201 & 0 & 0.0529 & 0.0916 & 0.0968 \\
\hline$\theta_{5}$ & $\theta_{6}$ & $\theta_{7}$ & $\theta_{8}$ & $\theta_{9}$ & $\theta_{10}$ \\
\hline 0.1187 & 0.1613 & 0.1819 & 0.3741 & 0.3935 & 1 \\
\hline
\end{tabular}

\section{Appendix B}

The optimal mechanism design is described by:

$$
\begin{gathered}
\max _{D R(\cdot), Y(\cdot)} E_{\theta}\left(U^{\mathrm{s}}(D R(\theta, L), Y(D R(\theta, L)), L)\right. \\
\left.+U^{\mathrm{d}}(D R(\theta, L), Y(D R(\theta, L)), \theta)\right) \\
U^{\mathrm{s}}(D R(\theta, L), Y(D R(\theta, L)), L) \\
=V^{\mathrm{s}}(L, D R(\theta, L))-Y(D R(\theta, L)) \geq 0 \\
U^{\mathrm{d}}(D R(\theta, L), Y(D R(\theta, L)), \theta) \\
=Y(D R(\theta, L))-C(\theta, d r) \geq 0 \\
U^{\mathrm{d}}(D R(\theta, L), Y(D R(\theta, L)), \theta) \\
\geq U^{\mathrm{d}}(D R(\tilde{\theta}, L), Y(D R(\tilde{\theta}, L)), \theta)
\end{gathered}
$$

Both the energy supplier and customers are assumed to be rational. An energy supplier will only provide compensation for customers at more critical locations where curtaining some load can reduce any hidden dangers to power grid security. Customers will not curtail load unless there is a positive incentive for them, so both customers and suppliers are subject to the individual rational constraint.

As mentioned above, $\theta$ is the parameter indicating customer willingness to curtail, and it is normalized as $0 \leq \theta \leq 1$, sorting the customers from the least willing to the most willing to curtail load. Hence, we have

$U^{\mathrm{d}}(D R(\underline{\theta}, L), Y(D R(\underline{\theta}, L)), \underline{\theta})=0$

Let

$$
\bar{U}^{\mathrm{d}}(\theta, L) \equiv \max _{\tilde{\theta}} U^{\mathrm{d}}(D R(\tilde{\theta}, L), Y(X(\tilde{\theta}, L)), \theta)
$$

From the individual rationality constraint for $\theta$, and the incentive compatibility constraint, we have

$$
\bar{U}^{\mathrm{d}}(\theta, L)=U^{\mathrm{d}}(D R(\theta, L), Y(D R(\theta, L)), \theta)
$$

According to the chain rule

$\frac{\partial \bar{U}^{\mathrm{d}}}{\partial \theta}=\frac{\partial U^{\mathrm{d}}}{\partial d r} \frac{\partial D R}{\partial \theta}+\frac{\partial U^{\mathrm{d}}}{\partial y} \frac{\partial Y}{\partial d r} \frac{\partial D R}{\partial \theta}+\frac{\partial U^{\mathrm{d}}}{\partial \theta}$
The energy supplier designs the special compensation scheme $Y(d r)$ for each customer, and the customers will self-select the optimal amount of demand response $d r$ to maximize their utility $U^{\mathrm{d}}(D R(\theta, L), Y(D R(\theta, L)), \theta)$, given by the point at which the first order condition is zero:

$\frac{\partial U^{\mathrm{d}}}{\partial d r}+\frac{\partial U^{\mathrm{d}}}{\partial y} \frac{\partial Y}{\partial d r}=0$

Then, it follows that

$$
\begin{aligned}
& \frac{\partial \bar{U}^{\mathrm{d}}}{\partial \theta}=\frac{\partial U^{\mathrm{d}}}{\partial \theta} \\
& \bar{U}^{\mathrm{d}}(\theta, L)=U^{\mathrm{d}}(\operatorname{DR}(\underline{\theta}, L), Y(D R(\underline{\theta}, L)), \underline{\theta}) \\
& \quad+\int_{\underline{\theta}}^{\theta} \frac{\partial U^{\mathrm{d}}}{\partial \theta}(D R(\tilde{\theta}, L), \tilde{\theta}) \mathrm{d} \tilde{\theta}
\end{aligned}
$$

From (B3)

$\frac{\partial U^{\mathrm{d}}}{\partial \theta}=-\frac{\partial C}{\partial \theta}$

Substitute (B12) into (B11)

$$
\begin{aligned}
\bar{U}^{\mathrm{d}}(\theta, L)=U^{\mathrm{d}} & (D R(\underline{\theta}, L), Y(D R(\underline{\theta}, L)), \underline{\theta}) \\
& -\int_{\underline{\theta}}^{\theta} \frac{\partial C}{\partial \theta}(D R(\tilde{\theta}, L), \tilde{\theta}) \mathrm{d} \tilde{\theta}
\end{aligned}
$$

And according to (B5), we can get

$\bar{U}^{\mathrm{d}}(\theta, L)=-\int_{\underline{\theta}}^{\theta} \frac{\partial C}{\partial \theta}(D R(\tilde{\theta}, L), \tilde{\theta}) \mathrm{d} \tilde{\theta}$

Furthermore, with (B2), (B3) and (B7), we have

$U^{\mathrm{s}}=V^{\mathrm{s}}-C-\bar{U}^{\mathrm{d}}$

Substitute (B14) and (B15) into (B1), we can obtain

$$
\begin{array}{r}
\max _{D R(\cdot)} \int_{\underline{\theta}}^{1}\left[V^{s}(D R(\theta, L))-C(D R(\theta, L), \theta)\right. \\
\left.+\int_{\underline{\theta}}^{\theta} \frac{\partial c}{\partial \theta}(D R(\tilde{\theta}, L), \tilde{\theta}) \mathrm{d} \tilde{\theta}\right] f(\theta) \mathrm{d} \theta
\end{array}
$$

where $F(\theta)=\int_{0}^{\theta} f(\tilde{\theta}) \mathrm{d} \tilde{\theta}$.

The first order derivative is zero, and the maximum $D R(\cdot)$ is where

$\frac{\partial V^{s}}{\partial D R}-\frac{\partial C}{\partial D R}+\frac{1-F(\theta)}{f(\theta)} \frac{\partial^{2} C}{\partial C \partial \theta}=0$

As mentioned above

$C(\theta, D R)=\frac{1}{2} D R^{2}+D R-D R \cdot \theta$

$V^{\mathrm{s}}=L \cdot D R(\theta)$

Substitute (B18) and (B19) into (B17), we have 
$D R(\theta, L)= \begin{cases}0 & 0 \leq \theta<1-\frac{L}{2} \\ 2 \theta+L-2 & 1-\frac{L}{2} \leq \theta<1\end{cases}$

From (B3), we have

$Y(D R(\theta, L))=U^{\mathrm{d}}(\theta, L)+C(X(\theta, L), \theta)$

Substitute (B20) into (B18), we have

$$
\begin{aligned}
C(\theta, D R) & =\frac{1}{2} D R^{2}+D R-D R \cdot \theta \\
& =\frac{1}{2}(2 \theta+L-2)^{2}+(2 \theta+L-2)-(2 \theta+L-2) \theta \\
& =\frac{1}{2} L^{2}+\theta L-L
\end{aligned}
$$

From (B14), we have

$$
\begin{aligned}
U^{\mathrm{d}}(\theta, L) & =-\int_{\underline{\theta}}^{\theta} \frac{\partial C}{\partial \theta}(D R(\tilde{\theta}, L), \tilde{\theta}) \mathrm{d} \tilde{\theta} \\
& =-\int_{\underline{\theta}}^{\theta}(-D R(\tilde{\theta}, L), \tilde{\theta}) \mathrm{d} \tilde{\theta} \\
& =\int_{\underline{\theta}}^{\theta}(D R(\tilde{\theta}, L), \tilde{\theta}) \mathrm{d} \tilde{\theta} \\
& =\int_{\underline{\theta}}^{\theta}(2 \tilde{\theta}+L-2) \mathrm{d} \tilde{\theta} \\
& =\theta^{2}-2 \theta+\theta L+\frac{1}{4} L^{2}-L+1
\end{aligned}
$$

Finally, substitute (B22) and (B23) into (B21), and we have

$$
Y(D R(\theta, L))=\left\{\begin{array}{lc}
0 & 0 \leq \theta<1-\frac{L}{2} \\
\theta^{2}-2 \theta+2 \theta L+\frac{3}{4} L^{2}-2 L+1 & \\
& 1-\frac{L}{2} \leq \theta<1
\end{array}\right.
$$

\section{References}

[1] Zhao C, Wang J, Watson JP et al (2013) Multi-stage robust unit commitment considering wind and demand response uncertainties. IEEE Trans Power Syst 28(3):2708-2717

[2] Rahiman FA, Zeineldin HH, Khadkikar V et al (2014) Demand response mismatch (DRM): concept, impact analysis, and solution. IEEE Trans Smart Grid 5(4):1734-1743

[3] Wu D, Aliprantis DC, Ying L (2012) Load scheduling and dispatch for aggregators of plug-in electric vehicles. IEEE Trans Smart Grid 3(1):368-376

[4] Vazquez S, Lukic SM, Galvan E et al (2010) Energy storage systems for transport and grid applications. IEEE Trans Ind Electron 57(12):3881-3895
[5] Tan KM, Ramachandaramurthy VK, Yong JY (2016) Integration of electric vehicles in smart grid: a review on vehicle to grid technologies and optimization techniques. Renew Sustain Energy Rev 53:720-732

[6] Zhou L, Li F, Gu C et al (2014) Cost/benefit assessment of a smart distribution system with intelligent electric vehicle charging. IEEE Trans Smart Grid 5(2):839-847

[7] Bahramirad S, Reder W, Khodaei A (2012) Reliability-constrained optimal sizing of energy storage system in a microgrid. IEEE Trans Smart Grid 3(4):2056-2062

[8] Department of Energy of the United States (2006) Benefits of demand response in electricity markets and recommendations for achieving them: a report to the United States congress pursuant to section 1252 of energy policy act of 2005. https:// energy.gov/oe/downloads/benefits-demand-response-electricitymarkets-and-recommendations-achieving-them-report. Accessed 10 February 2006

[9] Tellidou AC, Bakirtzis AG (2009) Demand response in electricity markets. In: Proceedings of 15 th international conference on intelligent system applications to power systems, Curitiba, Brazil, 8-12 November 2009, 6 pp

[10] Albadi MH, El-Saadany EF (2008) A summary of demand response in electricity markets. Electric Power Syst Res 78(11):1989-1996

[11] Singh SN, Østergaard J (2010) Use of demand response in electricity markets: an overview and key issues. In: Proceedings of 7 th international conference on the European energy market, Madrid, Spain, 23-25 June 2010, 6 pp

[12] Kim DM, Kim JO (2012) Design of emergency demand response program using analytic hierarchy process. IEEE Trans Smart Grid 3(2):635-644

[13] Zhong MM, Chen JQ, Wang K et al (2014) A centralized decision method for multi-time scale coordinated orderly power consumption. Power Syst Technol 38(22):70-77

[14] David AK, Wen FS (2001) Market power in electricity supply. IEEE Trans Energy Convers 16(4):352-360

[15] Wang BB, Sun YJ, Li Y (2015) Application of uncertain demand response modeling in power-score incentive decision. Autom Electr Power Syst 39(10):93-99

[16] Niu WJ, Li Y (2014) Uncertain optimization decision of interruptible load in demand response program. In: Proceedings of IEEE innovative smart grid technologies-Asia (ISGT Asia), Kuala Lumpur, Malaysia, 20-23 May 2014, pp 675-679

[17] Leonid H, Stanley R (2006) Designing economic mechanisms. Cambridge University Press, England

[18] Fahrioglu M, Alvarado FL (1999) Designing cost effective demand management contracts using game theory. In: Proceedings of IEEE power engineering society 1999 winter meeting, New York, USA, 31 January-4 February 1999, pp 427-432

[19] Fahrioglu M, Alvarado FL (2000) Designing incentive compatible contracts for effective demand management. IEEE Trans Power Syst 15(4):1255-1260

[20] Chen Z, Wu L, Fu Y (2012) Real-time price-based demand response management for residential appliances via stochastic optimization and robust optimization. IEEE Trans Smart Grid 3(4): 1822-1831

[21] Aalami HA, Parsa MM, Yousefi GR (2010) Demand response modeling considering interruptible/curtailable loads and capacity market programs. Appl Energy 87(1):243-250

[22] Kirschen DS, Strbac G, Cumperayot P et al (2000) Factoring the elasticity of demand in electricity prices. IEEE Trans Power Syst 15(2):612-617

[23] Su CL, Kirschen D (2009) Quantifying the effect of demand response on electricity markets. IEEE Trans Power Syst 24(3):1199-1207 
[24] Wu L (2013) Impact of price-based demand response on market clearing and locational marginal prices. IET Gen Trans Dist 7(10): 1087-1095

[25] Ngan HW (2010) Electricity regulation and electricity market reforms in China. Energy Policy 38:2142-2148

[26] Fahrioglu M, Alvarado FL (2001) Using utility information to calibrate customer demand management behavior models. IEEE Trans Power Syst 16(2):317-322

[27] Jian XH, Zhang L, Miao XF et al (2018) Designing interruptible load management scheme based on customer performance using mechanism design theory. Electr Power Energy Syst 95:476-489

[28] Madani R, Ashraphijuo M, Lavaei J (2016) Promises of conic relaxation for contingency-constrained optimal power flow problem. IEEE Trans Power Syst 31(2):1297-1307

[29] Wood AJ, Wollenberg BF (1996) Power generation, operation, and control, 2nd edn. Wiley, New York

[30] Carrion M, Arroyo JM (2006) A computationally efficient mixed-integer linear formulation for the thermal unit commitment problem. IEEE Trans Power Syst 21(3):1371-1378

[31] Ostrowski J, Anjos MF, Vannelli A (2012) Tight mixed integer linear programming formulations for the unit commitment problem. IEEE Trans Power Syst 27(1):39-46

[32] Arroyo JM, Conejo AJ (2000) Optimal response of a thermal unit to an electricity spot market. IEEE Trans Power Syst 15(3):1098-1104

[33] Bouffard F, Galiana FD (2004) An electricity market with a probabilistic spinning reserve criterion. IEEE Trans Power Syst 19(1):300-307

[34] Floudas CA (1955) Nonlinear and mixed-integer optimization: fundamentals and applications, 1st edn. Oxford University Press, New York

[35] Wang J, Shahidehpour M, Li Z (2008) Security-constrained unit commitment with volatile wind power generation. IEEE Trans Power Syst 23(3):1319-1327

[36] Samimi A, Nikzad M, Mohammadi M (2016) Real-time electricity pricing of a comprehensive demand response model in smart grids. Int Trans Electr Energ Syst 27(3):1-15

[37] Wu L, Shahidehpour M, Li T (2007) Stochastic security-constrained unit commitment. IEEE Trans Power Syst 22(2):800-811
Yumin ZHANG received her M.E. degree from Liaoning University of Technology, Jinzhou, China, in 2013. Currently, she is pursuing her Ph.D. degree in Shandong University, Jinan, China. Her research interests include power system operation and control, electricity market and demand response.

Xueshan HAN received the Ph.D. degree from Harbin Institute of Technology, Harbin, China, in 1994, in electrical engineering. From 1998 to 2000, he was a research fellow in Nanyang Technological University. He is now a professor and serving as vice dean of the School of Electrical Engineering, Shandong University, Jinan, China. His research interests include energy system forecasting, power system economic dispatch, electricity market design, power system operation optimization and control.

Li ZHANG received the B.E. degree from Tianjin University, Tianjin, China, in 1989, and the M.E. and Ph.D. degrees from the Shandong University of Technology and Shandong University, Jinan, China, in 1992 and 2006, respectively. She is currently an associate professor at Shandong University, Jinan, China. Her research interests include power system operation and control and electricity market.

Bo XU received the B.E. and Ph.D. degrees from Shandong University, Jinan, China, in 2010 and 2016, respectively. He is now a postdoctoral fellow in Department of Electrical Engineering, Tsinghua University, Beijing, China. His research interests include power system economic dispatch, power system condition-based maintenance.

Mingqiang WANG received the Ph.D. degree from Nanyang Technological University, Singapore, in 2012. Currently he is an associate professor at the School of Electrical Engineering, Shandong University, Jinan, China. His research interests are power system economic operation and microgrid.

Ming YANG received the Ph.D. degree in electrical engineering from Shandong University, Jinan, China, in 2009. Currently, he is an associate professor at Shandong University. His research interests include energy system forecasting, power system analysis and power system operation optimization. 